\title{
Neuronal Populations Stained with the Monoclonal Antibody Cat-301 in the Mammalian Cerebral Cortex and Thalamus
}

\author{
S. H. C. Hendry, ${ }^{1}$ E. G. Jones, ${ }^{1}$ S. Hockfield, ${ }^{2}$ and R. D. G. McKay ${ }^{3}$ \\ 'Department of Anatomy and Neurobiology, University of California, Irvine, Irvine, California 92717, ${ }^{2}$ Section of \\ Neuroanatomy, Yale University School of Medicine, New Haven, Connecticut 06510, and ${ }^{3}$ Department of Biology, \\ Massachusetts Institute of Technology, Cambridge, Massachusetts 02139
}

The monoclonal antibody Cat-301 was used to examine neurons in the cerebral cortex and dorsal thalamus of several mammalian species, including Old World monkeys, cats, bush babies, guinea pigs, and rats. In each species, subpopulations of cortical and thalamic neurons are stained along the surfaces of their somata and proximal dendrites. Cat-301positive cortical neurons include specific groups of pyramidal cells (e.g., corticospinal but not corticobulbar or callosal neurons in the monkey sensory-motor areas) and certain GABA-immunoreactive nonpyramidal cells. In the thalamus, the relay neurons projecting to the cortex and not the intrinsic neurons are stained.

The Cat-301-positive neurons are nonhomogeneously distributed in the cat and monkey cortex and thalamus. In the cortex, they are densely packed in 2 bands that in most areas include layers III and $\mathrm{V}$, but that in primary sensory areas include layers IV and VI. Because the density of stained neurons, their distribution, and the intensity of their staining vary among cortical areas, the borders between neighboring areas can often be detected by the differences in Cat-301 staining. Broader, regional differences are also readily apparent, for areas in the parietal and occipital lobes contain large numbers of intensely stained cells, but most areas in the frontal and temporal lobes contain fewer, more lightly stained neurons. The same broad differences are seen within the thalamus: only those nuclei reclprocally connected with intensely stained cortical areas contain large numbers of Cat-301-positive neurons.

Differences among species include variations in cell density and distribution when a given cortical area or thalamic nucleus is compared between cats and monkeys. Greater differences are seen among the other species. Immunoreactive neurons in the cerebral cortex are sparse and lightly stained in guinea pigs, are restricted to the hippocampal formation in rats, and are very rare and isolated in bush babies. Similarly, Cat-301-positive thalamic neurons are restricted to only one or 2 nuclei in the guinea pig and rat and are extremely rare in the bush baby.

Cat-301 stains organized groups of neurons in the cat and monkey cortex and thalamus. In addition to the laminar or-

Received Feb. 4, 1987; revised June 29, 1987; accepted Aug. 13, 1987.

This work was supported by Grants EY 06432, FY 06517, NS 21377, and NS 07650 from the National Institutes of Health. S.H.C.H. is an Alfred P. Sloan Foundation Fellow.

Correspondence should be addressed to Stewart Hendry at the above address. Copyright (c) 1988 Society for Neuroscience $0270-6474 / 88 / 020518-25 \$ 02.00 / 0$ ganization of stained cells in all cortical areas (see above), the Cat-301-positive neurons of monkey areas 17 and 18 are grouped into radial arrays. In area 17 , clusters of stained cells are present in layers above and below layer IVC. These clusters lie at the centers of ocular dominance columns, within patches stained for cytochrome oxidase (CO). Most of these cells are also GABA-immunoreactive. In area 18, bands of immunoreactive cells are present in layers III-V, where they are found to coincide with $\mathrm{CO}$ bands in this area. In the cat and monkey dorsal lateral geniculate nuclei, the distribution of Cat-301-positive neurons resembles the distribution of a particular functional class (the Y-like neurons), while in the monkey ventral posterior medial nucleus, the immunoreactive cells form elongated rods that interdigitate with the terminations of afferents from the principal trigeminal nucleus.

These data indicate that neurons expressing the Cat-301 antigen(s) are a morphologically and chemically heterogeneous population of cells that are present in the cortex and thalamus of 5 mammalian species. Within a broader pattern of distribution, groups of these cells form units in which neurons are related by common functional or connectional attributes.

Neuronal diversity is the hallmark of the mammalian CNS, and nowhere is this more apparent than in the cerebral cortex. There, neuronal diversity has been described in morphological terms for the last century (Golgi, 1883; Ramon y Cajal, 1891; Retzius, 1893; Kölliker, 1896) and, at the present time, as many as 10 basic neuronal types are recognized by most workers (e.g., Valverde, 1971; Lund, 1973; Jones, 1975; Peters and Regidor, 1981; Fairén et al., 1984; Peters and Jones, 1984). The characteristics used for classifying neuronal types include such well-established parameters as somal size, dendritic architecture, and the nature of the axonal ramification. Often, morphological distinctions made in this way have proven to have physiological correlates (Gilbert and Wiesel, 1979; Parnavelas et al., 1983; Martin and Whitteridge, 1984), although it is still by no means established that, for example, all cells with different types of receptive fields in a sensory area of the cortex are morphologically distinct and vice versa.

Diversity in the cerebral cortex is also reflected in its division into cytoarchitectonic fields. Distinctive characteristics of these fields include variations in size, laminar distribution, and packing density of neurons, connections, and physiological properties (e.g., Hubel and Wiesel, 1962, 1968; Gilbert and Kelly, 1975; Lund et al., 1975; Gilbert, 1977; Jones and Wise, 1977), 
and often an organization into detectable functional units, or "modules," that in some cases have demonstrable anatomical correlates (Wiesel et al., 1974; Jones et al., 1975, 1979; LeVay et al., 1975; Horton and Hubel, 1981; Livingstone and Hubel, 1984a). Cytoarchitectonic and functional parcellations of similar type also exist in other areas of the CNS such as the thalamus (see Jones, 1985).

Neuronal diversity in the CNS may also be reflected in molecular characteristics. One obvious indication of this is the expression of a neurotransmitter or of a class of receptors (Houser et al., 1983; Emson and Hunt, 1984; Streit, 1984; Wamsley, 1984; Jones and Hendry, 1986). Another is the expression of intracellular or surface molecules that may have little to do with the process of neuronal transmission, but may identify unique neuronal subpopulations (Barnstable, 1980; Zipser and McKay, 1981; Hockfield and McKay, 1983a; McKay et al., 1983). Such molecules may even identify subclasses of neurons that on all other counts appear the same (Hockfield et al., 1983; Johansen et al., 1985). Estimates of the extremely large numbers of unique mRNAs expressed by vertebrate central nervous system cells (Chikaraishi et al., 1983; Hahn et al., 1983) would suggest that the number of such molecules yet to be discovered is very high.

One approach to identifying such molecules is the production of monoclonal antibodies, made against relatively impure mixtures of nervous tissue and used to localize the molecules immunocytochemically. This strategy has yielded productive results in the detection of neuron-specific antigens in the nervous system of the leech (Zipser and McKay, 1981; Zipser, 1982; Hockfield and McKay, 1983b; McKay et al., 1983), in the vertebrate retina (Barnstable, 1980; Lemmon and Gottlieb, 1982; Young and Dowling, 1984; Fry et al., 1985), and in various parts of the mammalian CNS (Hawkes et al., 1982; McKay and Hockfield, 1982; Sternberger et al., 1982; Levitt, 1984). Subsequent work will necessitate the isolation and identification of these antigens, as well as the characterization in detail of the neuronal populations specified by each. The present study is devoted to the second of these 2 lines of research, and deals with the distribution and the morphological, connectional, and neurotransmitter-related distinctions and similarities among neurons stained with a monoclonal antibody, Cat-301, in the cerebral cortex and thalamus of several mammalian species. Preliminary reports on certain aspects of this investigation have already appeared (Hendry et al., 1983a, 1984a; Hockfield et al., 1983; Jones et al., 1983).

\section{Materials and Methods}

The production of Cat-301 and the procedure for immunocytochemical staining have been described elsewhere (McKay and Hockfield, 1982; Hockfield and McKay, 1983a; Hendry et al., 1984a). Twelve cynomolgus monkeys (Macaca fascicularis), 7 domestic cats, 5 Wistar rats, 3 guinea pigs (Cavia porcellus), and 2 bush babies (Galago senegalensis) were perfused through the heart with $4 \%$ paraformaldehyde or with $4 \%$ paraformaldehyde and $0.1 \%$ glutaraldehyde, both in $0.1 \mathrm{~m}$ phosphate buffer $(\mathrm{pH} 7.4$ ). Blocks of the cerebral cortex and diencephalon were infiltrated with buffered $30 \%$ sucrose solution, cut on a freezing microtome at 10,25 , or $40 \mu \mathrm{m}$ and prepared for immunocytochemistry. For every series of sections stained with Cat-301, additional sections were processed with normal mouse serum as a replacement for the monoclonal antibody to serve as a control for the nonspecific binding of mouse immunoglobulins (Igs) and of labeled anti-mouse Igs. No control section displayed staining of either the cell surface or of the neuropil comparable to that seen in Cat-301-stained sections.

The whole cerebral cortex of each species was examined either by staining a 1 -in- 10 series through its entirety (rats, guinea pigs, cats, and bush babies) or by first dissecting the cortex into lobes and then cutting and staining a regular series of sections through each lobe (monkeys). The whole thalamus was examined in all species. Selected sections through both the thalamus and cortex were counterstained with thionin.

Three monkeys and the members of the other species were normal. The following experiments were performed on the other 9 monkeys:

1. Retrograde transport experiments involving sensory-motor cortex. Seven of the monkeys were injected with the fluorescent dye, Fast blue $(8 \% \mathrm{wt} / \mathrm{vol}$ in distilled water). Two received multiple injections of $0.3-$ $0.5 \mu$ l unilaterally in the spinal cord at levels corresponding to the fourth to eighth cervical segments $(\mathrm{C} 4-\mathrm{C} 8), 2$ received injections $(0.1-0.2 \mu \mathrm{l})$ into the cuneate and gracile nuclei, and 3 received multiple injections $(0.2 \mu \mathrm{l}$ each $)$ unilaterally into the pre- or postcentral gyrus. These animals were allowed to survive from 10 to $14 \mathrm{~d}$ before they were killed. Sections of the sensory-motor cortex were processed for Cat-301 using a fluorescein isothiocyanate (FITC)-conjugated goat anti-mouse Ig (Cappel). They were examined with a fluorescence microscope equipped with FITC and broadband ultraviolet filters. The thalamus in those animals receiving injections into the pre- or postcentral gyrus was also processed for the simultaneous detection of retrogradely transported fast blue and immunostaining by Cat- 301 .

2. Anterograde transport experiments involving the thalamus. In 2 of the monkeys, the principal nucleus of the brain stem trigeminal complex was injected with $0.2 \mu \mathrm{l}$ of HRP (type VI; Sigma), $50 \% \mathrm{wt} / \mathrm{vol}$ in normal saline. The nucleus was localized by first recording single-unit responses to tactile stimulation of the mouth and face. Following a survival of 2 d, the animals were perfused with $4 \%$ paraformaldehyde and $0.1 \%$ glutaraldehyde. Sections through the thalamus contralateral to the injection were cut at $40 \mu \mathrm{m}$ and collected serially. Every other section was processed for HRP histochemistry by the method of DeOlmos et al. (1978). The adjacent sections were pretreated with $1 \%$ hydrogen peroxide in $50 \%$ methanol to remove peroxidase activity and were then processed for Cat-301 staining. The 2 sets of sections were mounted serially and adjacent sections were compared.

3. Experiments on the primary visual cortex of monkeys. One eye was surgically removed from 6 of the monkeys 1-2 weeks before they were killed. During postfixation and sucrose infiltration, both occipital lobes of these monkeys and of the other 6 monkeys were flattened and sectioned at $30 \mu \mathrm{m}$ parallel to the opercular surface. Every other section was stained for cytochrome oxidase (CO) activity by the method of Wong-Riley (1979). The adjacent sections were stained with Cat-301; the sections of the 2 series were mounted in alternating sequence and were analyzed by superimposing the same radially oriented blood vessels in the adjacent sections.

Two other procedures were performed on normal animals:

1. Double immunocylochemical staining experiments. Three of the monkeys and 2 of the cats were prepared for simultaneous immunocytochemical staining with Cat-301 and for either GABA, somatostatin (SRIF), neuropeptide Y (NPY), or cholecystokinin octapeptide (CCK). Sections through the cortex and thalamus were incubated in Cat-301 and anti-GABA (diluted 1:1500; ImmunoNuclear), anti-SRIF (1:500; Dako), anti-NPY (1:1000; Amersham), or anti-CCK (1:500; ImmunoNuclear), washed and incubated in both FITC-conjugated goat antimouse Ig (1:100; Cappel) and tetramethyl rhodamine isothiocyanate (TRITC)-conjugated goat anti-rabbit Ig (1:200; Cappel). The sections were examined by fluorescence microscopy using FITC and TRITC filter packs. Control sections in which the anti-GABA or the anti-peptide antisera were replaced with antisera preadsorbed with an excess of antigen showed only Cat-301 staining. The anti-GABA was adsorbed with $20 \mu \mathrm{g} / \mathrm{ml}$ of GABA, and the anti-CCK, -SRIF and -NPY with $10 \mu \mathrm{g} /$ $\mathrm{ml}$ of the respective synthetic peptides (Peninsula Labs and Bachem).

2. Counts of neurons. The number of Cat-301-positive neurons in 100- $\mu \mathrm{m}$-wide traverses through several cortical areas was determined for each species. Similar counts were also made in the dorsal thalamus of cats and monkeys. Two methods were used. In one, $10-\mu \mathrm{m}$-thick frozen sections were stained immunocytochemically and counterstained with thionin. At least 4 sections through an area were counted from the 2 members of each species that displayed the most intense staining, judged qualitatively. In the other method, $40-\mu \mathrm{m}$-thick sections cut on a Vibratome were stained, osmicated, and embedded in plastic. Blocks were cut at $2 \mu \mathrm{m}$ on a Porter-Blum MT2B ultramicrotome and stained with methylene blue and Azur II. In both the frozen and plastic sections, all Cat-301-immunoreactive neurons and nonimmunoreactive neurons possessing a stained nucleus were counted.

Counts were also made from $10-\mu \mathrm{m}$-thick frozen sections that had been prepared for simultaneous immunofluorescent detection of both 

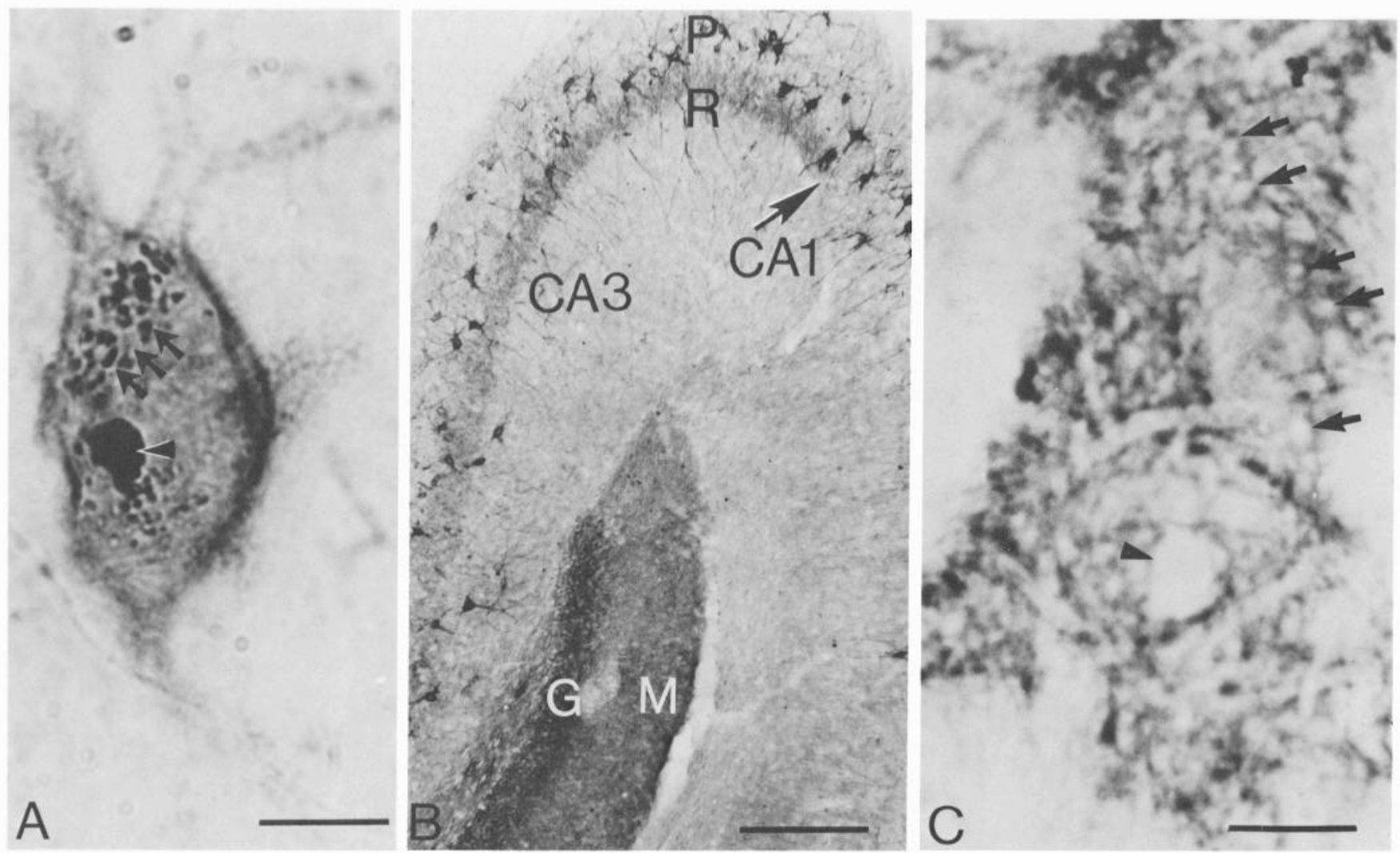

Figure 1. Staining of cortical neurons with Cat-301. A, Neuron in guinea pig cerebral cortex containing numerous small Cat-301-positive "packets" (arrows) and a large stained region (arrowhead) within its cytoplasm. In addition to the cytoplasmic staining, the cell is also stained along its surface. Bar, $5 \mu \mathrm{m}$. B, Cat-301 staining of the rat hippocampus. A small number of cell bodies in stratum pyramidale $(P)$ and stratum radiatum $(R)$ of fields $C A 1$ and $C A 3$ are intensely stained. In addition, the neuropil of the inner portion of stratum radiatum is stained in CA3, but not in CA1, so that the border between these fields (arrow) is apparent. Intense staining of neuropil is also found in the molecular layer ( $M$ ) and in the granule cell layer $(G)$ of the dentate gyrus. Bar, $80 \mu \mathrm{m}$. $C$, Staining of a pyramidal cell in layer $\mathrm{V}$ of monkey area 4 . The plane of the section grazes the surface of the soma, producing an en face view. Along the surface of the soma, dark Cat-301 staining surrounds numerous small, circular regions (arrows point to examples) and other, more elongated profiles that have been left unstained. The large, unstained region (arrowhead) is where the plane of section has cut through the cell surface, presumably at the origin of a dendrite. Bar, $15 \mu \mathrm{m}$.

GABA and Cat-301. Counts were made of single- and double-labeled cells in $100-\mu \mathrm{m}$-wide traverses through the sensory-motor, parietal, and primary visual areas of the 2 cats and the 3 monkeys prepared for fluorescent immunostaining. Counts were restricted to neurons with somal diameters of more than $8 \mu \mathrm{m}$ so as to reduce the frequency of partial-cell counting.

\section{Results}

Neurons in the cerebral cortex and thalamus of rats, guinea pigs, cats, bush babies, and monkeys are stained with Cat-301. In these species, a common pattern of staining of individual neurons is seen, but the numbers and types of immunoreactive neurons are greatest in cats and monkeys, and their distributions are the most complex. Hence, for the various features of Cat-
301 immunostaining, cats and monkeys will be described in detail and will be compared only briefly to the other species.

\section{Neuronal staining with Cat-301}

As reported previously for brain stem and spinal neurons(McKay and Hockfield, 1982; Hockfield and McKay, 1983a), the staining of cortical and thalamic cells with Cat-301 is primarily on the surfaces of neurons, where it outlines their somata and dendrites. The staining has a lattice-like appearance, and when the surface of a Cat-301-positive neuron is viewed en face, it appears fenestrated, with thin immunoreactive bands surrounding circular, unstained regions (Fig. 1C). In addition to the staining of cell surfaces, which is present in each species, Cat-301 stains cyto-

\begin{tabular}{|c|c|c|c|c|c|c|}
\hline \multicolumn{4}{|l|}{ Cat } & \multicolumn{3}{|l|}{ Monkey } \\
\hline Area & Cat-301 & Total & $\%$ & Cat-301 & Total & $\%$ \\
\hline 4 & $57.8 \pm 8.9$ & $309.2 \pm 23.7$ & $18.7 \pm 1.6$ & $32.4 \pm 4.9$ & $319.8 \pm 30.1$ & $10.3 \pm 0.9$ \\
\hline $1-2$ & $61.3 \pm 9.0$ & $337.4 \pm 30.1$ & $18.2 \pm 1.4$ & $34.8 \pm 5.1$ & $331.4 \pm 29.6$ & $10.4 \pm 1.2$ \\
\hline 17 & $62.7 \pm 7.8$ & $326.7 \pm 37.4$ & $19.2 \pm 1.5$ & $67.3 \pm 5.7$ & $667.4 \pm 49.3$ & $10.1 \pm 1.1$ \\
\hline
\end{tabular}




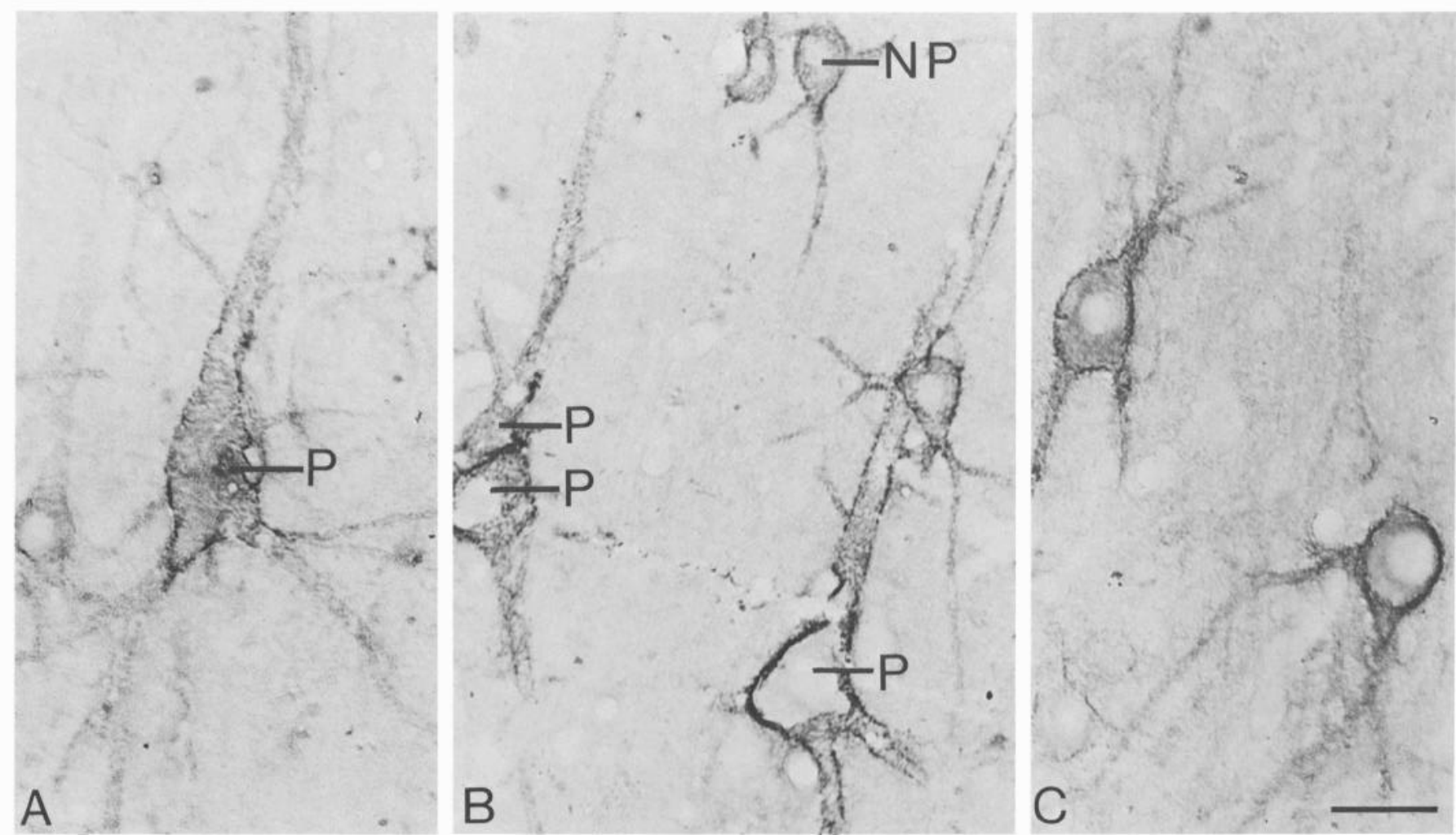

Figure 2. Cat-301-positive neurons in monkey sensory-motor cortex. $A$, Large neuron in layer $\mathrm{V}$ of area 4, possessing a triangular soma, a prominent apical dendrite, and a system of basal dendrites. By these features, the neuron can be classified as a pyramidal cell. $B$, Cat-301-positive cells in layer $\mathrm{V}$ of area 4 . Three cells possess the features of pyramidal cells $(P)$, while 2 others, with smaller, more oblong somata and multipolar dendritic arrangements appear to be nonpyramidal cells $(N P)$. C, Cat-301-positive cells in layer IIIB of area 4 . The round somata and multipolar dendritic organization of these neurons indicate they are nonpyramidal cells. Bar: $30 \mu \mathrm{m}(A, B) ; 40 \mu \mathrm{m}(C)$.

plasmic structures in the neurons of the guinea pig cortex, where densely immunoreactive profiles are present in the somata and dendrites of many surface-stained cells (Fig. 1A). This intracellular staining can be detected only within neurons that also display the typical Cat-301 staining on their surfaces.

Many cortical areas and thalamic nuclei that contain Cat301-positive cell somata also display a diffuse, yet dense, staining of parts of the neuropil. This neuropil staining varies in intensity among areas and nuclei, and while it is generally greatest in areas of cat cortex, it is also intense in specific areas of monkey cortex (e.g., layer VI of area 17) and rat cortex (e.g., the granule and molecular layers of the dentate gyrus; Fig. $1 B$ ).

\section{Cerebral cortex}

Numbers of cells stained in cortex. Although large numbers of Cat-301-positive neurons are present in the neocortex of cats and monkeys, only a fraction of the total neuronal population in any area of cortex is Cat-301-positive, and the number of immunoreactive neurons varies from area to area. Table 1 shows the number of Cat-301-positive neurons in a $100-\mu \mathrm{m}$-wide traverse through each of the 3 most densely stained areas, 4, 1-2, and 17. The greatest number of Cat-301-positive cells is found in areas 4 and 17 of cats, where 57-61 neurons are present within $100-\mu \mathrm{m}$-wide columns through the thickness of the cortex. The number of Cat-301-positive cells is considerably smaller than the total number of neurons counted in the same sections (Table 1). These figures indicate that, in the cat, approximately $20 \%$ of the neurons in areas 4, 1-2, and 17 are Cat-301-positive, but that in the monkey only $10 \%$ of the neurons are Cat-301-pos- itive. This lower percentage in monkey cortex is found for each area examined, including area 17, where the number of stained neurons is the same as in cats, but where the total number of neurons is far greater in the monkey (Table 1).

Classes of cortical neurons stained with Cat-301. Throughout the neocortex the Cat-301-positive neurons make up a heterogeneous population, with stained cells of different morphologies and sizes present within any cytoarchitectonic area. Two broad types of cells are immunoreactive. One has a large triangular-shaped soma and a single, prominent apical dendrite stained for approximately $100 \mu \mathrm{m}$ (Fig. 2, $A, B$ ). Multiple large, stained processes also emerge from the base of the soma (Fig. $2 A$ ). These are the morphological features of pyramidal neurons. The second prominent class of Cat-301-positive neurons has a rounded soma of variable size that gives rise to several stained processes (Fig. 2, B, C). The processes are all of equal size and emerge from any point along the surface of the soma, but for the larger-sized somata they most commonly arise from the upper and lower poles and adopt a vertical orientation (Fig. 2C). The processes are frequently stained for a distance of $200-300$ $\mu \mathrm{m}$, over which they divide once or twice. These are the morphological features of nonpyramidal cells, and the somal and dendritic features of the larger type are those of the large basket cell (Marin-Padilla, 1969; Jones, 1975; DeFelipe et al., 1986).

Combined retrograde transport and immunocytochemical experiments confirm that some pyramidal cells are Cat-301-positive and indicate that the staining is specific for certain pyramidal cell subpopulations. Within the monkey sensory-motor cortex, corticospinal neurons are Cat-301-positive (Fig. 3; see 


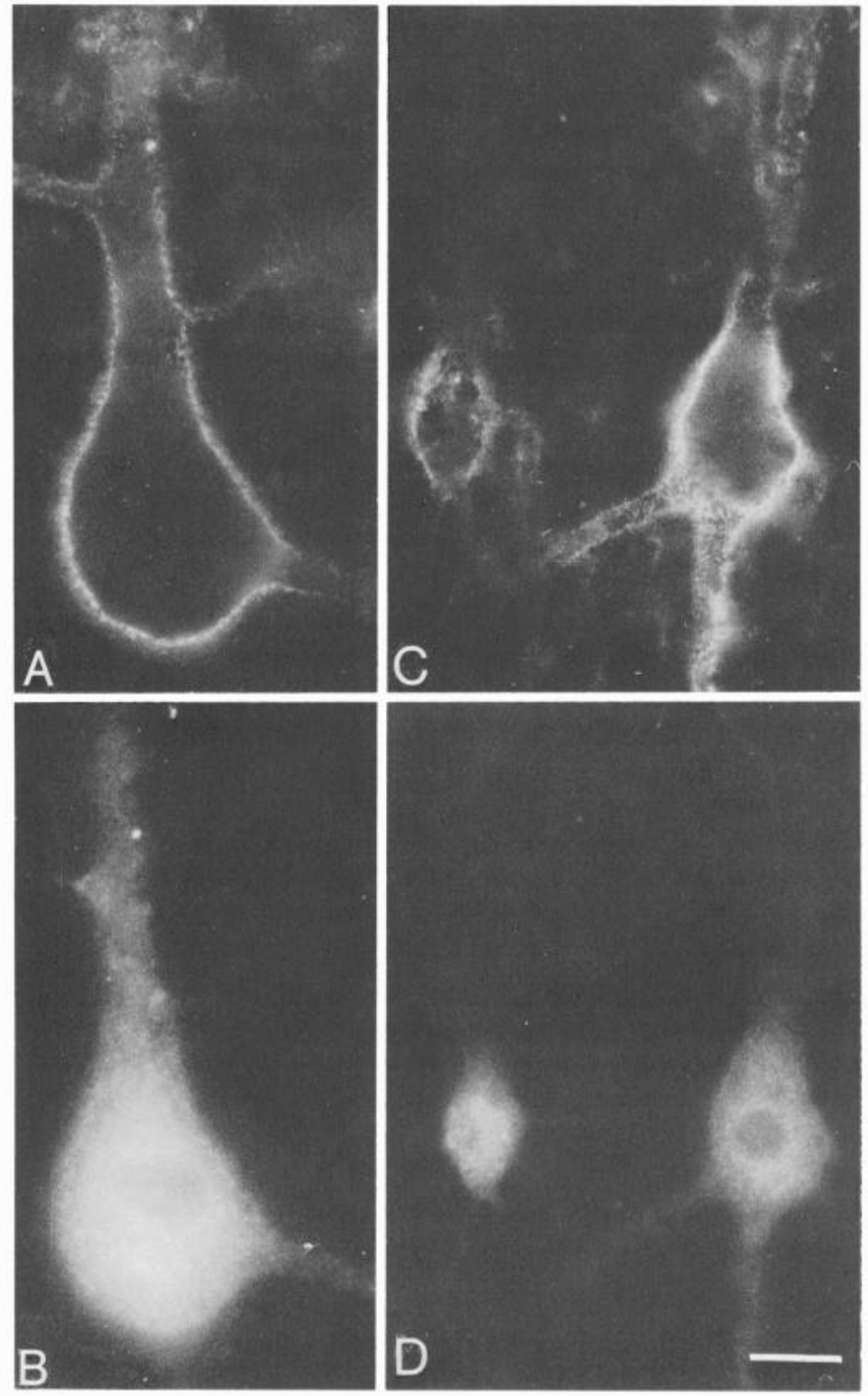

Figure 3. Cat-301 staining of corticospinal neurons in monkey area 4. $A, B$, Fluorescence photomicrographs of a large layer $\mathrm{V}$ pyramidal cell (Betz cell) that is immunostained with Cat-301 $(A)$ and also labeled with Fast blue following retrograde transport from an injection in the spinal cord $(B) . C, D$, Two cells of different sizes in layer $\mathrm{V}$ are stained with Cat-301 (C); both are retrogradely labeled following injections of Fast blue into the spinal cord $(D)$. Bar, $15 \mu \mathrm{m}$.

also Hockfield et al., 1983). Injections of Fast blue into the monkey cervical spinal cord (including the dorsal and ventral horns and the corticospinal tracts at levels C3-C8) label pyramidal neurons in layer $\mathrm{V}$ throughout the forelimb, trunk, and hindlimb representations in area 4 and in the first somatic sensory area (SI). Corticospinal cells of all sizes are Cat-301-positive (Fig. 3, $A-D$ ), including the extremely large Betz cells, with somata that approach $50 \mu \mathrm{m}$ in diameter (Fig. 3, C, D). Scattered among the Betz cells in layer $\mathrm{V}$ is a small but consistent population of Cat-301-positive pyramidal cells left unlabeled by the spinal cord injections.

By contrast with corticospinal neurons, cortical cells labeled by Fast blue injections into the dorsal column nuclei are not Cat-301-positive. Following these injections, fast blue-labeled and Cat-301-positive cells often lie side-by-side in the same section through the sensory-motor cortex, but no cell is double- labeled. Similarly, layer III pyramidal cells labeled by fast blue injections into the contralateral sensory-motor areas are not stained by Cat-301, even though large numbers of immunoreactive neurons are present in this layer.

Double-staining by immunocytochemistry confirms that nonpyramidal cells are also Cat-301-positive, since many of the Cat-301-positive neurons in the cat and monkey cortex are immunoreactive for the amino acid neurotransmitter GABA, a substance detected only in nonpyramidal neurons. The fluorescence photomicrographs in Figures 4 and 5 are taken from the same double-labeled sections through monkey area 4 and cat area 17. Many nonpyramidal somata that display cytoplasmic GABA immunostaining are stained along their surfaces by Cat301 (Figs. 4, $E, F$; 5). Adjacent pyramidal cells that are covered by GABA-positive terminals, but are themselves not stained for GABA, are also Cat-301-positive (Fig. 4, $A-D$ ). Quantitative analyses of neurons in area 17 of cat cortex reveal that almost $80 \%$ of the GABA-positive neurons are stained with Cat-301; these double-labeled neurons make up over two-thirds of the total Cat-301-positive population in area 17 (see Fig. 5). Similar proportions exist within the sensory-motor and parietal association areas of cat cortex. In the monkey cerebral cortex, doublelabeled cells include approximately half the GABA population and $40-60 \%$ of the Cat-301 population. Thus, the Cat-301positive and GABA-positive populations overlap, but neither is completely contained within the other.

In monkey cerebral cortex, a small population of Cat-301positive nonpyramidal cells in layer VI and in the white matter immediately deep to it also displays immunoreactivity for one of the neuropeptides, CCK, SRIF, or NPY. However, most of the peptide-immunoreactive neurons in these and other layers could not be stained with Cat-301.

Laminar organization of Cat-301-positive neurons and variability among areas. In both cat and monkey cerebral cortex, Cat-301-positive cells are distributed unevenly across layers. Although present in all layers, the immunostained cells are densely packed in some and are sparse in others; this laminar distribution varies from area to area. Cat-301-positive cells in both the cat and monkey neocortex adopt a basically bilaminar distribution, with large numbers present in 2 bands: a superficial band that usually includes layer IIIB and a deep band that is largely coextensive with either layer VB or VI (Fig. 6). Areal differences in the cat cortex arise from variations in the width of the superficial band, differences in the density of stained cells in the 2 bands, and the varying degree to which a third, deep band is stained. Such differences are apparent between areas 17 and 18 of cat visual cortex (Fig. $7 \mathrm{~A}$ ). The intensely stained superficial band of cells in area 17 is wide and includes both layers IIIB and IV, while in area 18 the band contains fewer Cat-301-positive cells and includes only layer IIIB. The stained cells also form a relatively dense band in layer VI of area 17, but this is reduced in area 18. Although the staining differences are most dramatic between areas 17 and 18, similar changes in staining patterns are evident between other areas of cat cortex (Fig. $7 A$ ), thus marking architectonic borders.

In the monkey cerebral cortex, it is mainly a difference in the depths of the Cat-301-stained bands that serves to mark the borders between areas. As in the cat, most areas of monkey cortex possess relatively dense bands of cells in layers IIIB and $\mathrm{V}$, but in some areas stained cells form major bands that include layer IV, or some component of that layer, and layer VI (Figs. $6, C-E ; 7 B)$. Such a distribution is found in area 17 , where 


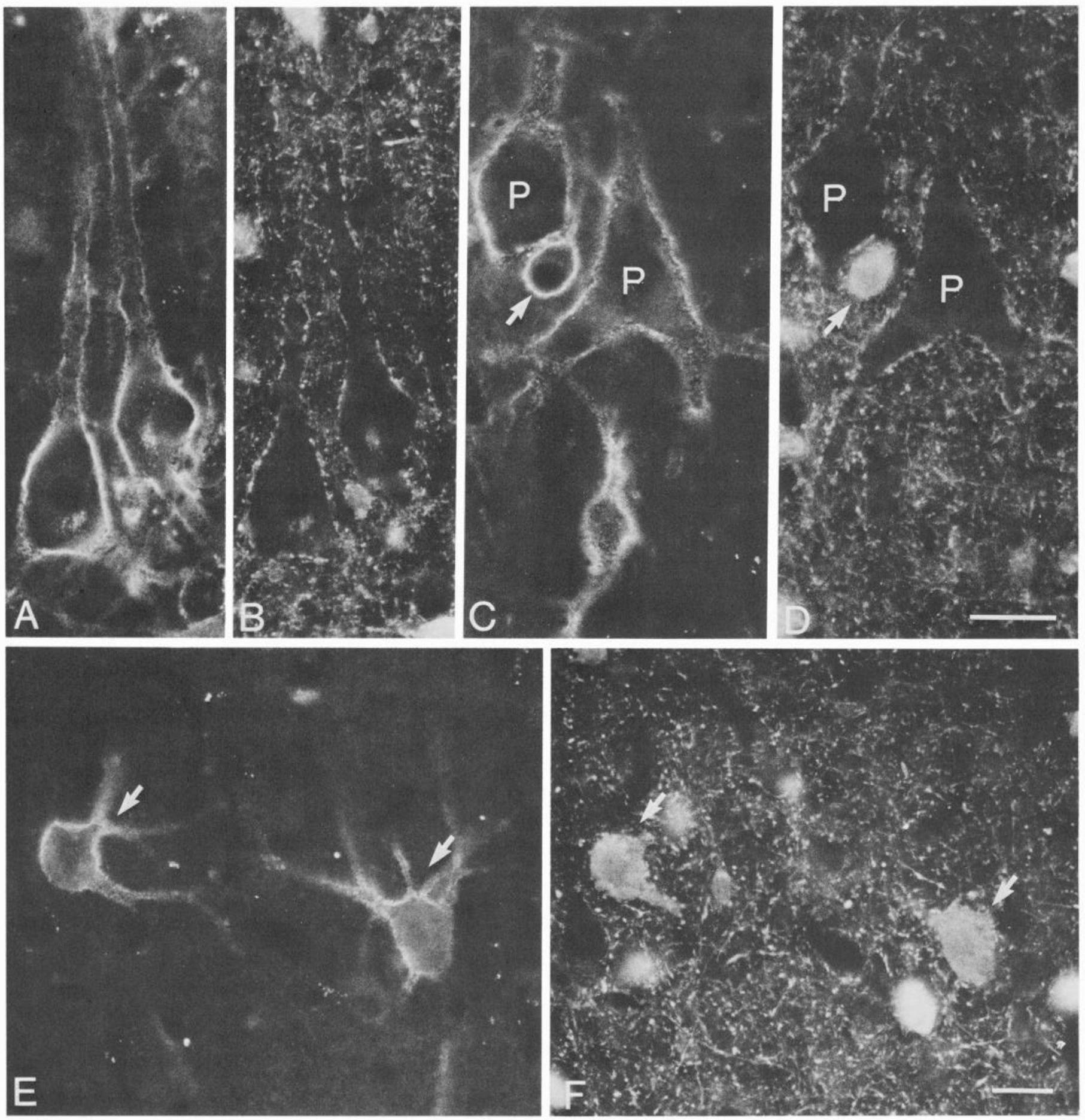

Figure 4. Correlation of Cat-301 and GABA immunostaining of neurons in monkey area 4. A, B, Cat-301 staining $(A)$ of 2 large layer V pyramidal cells (Betz cells). The cells display no GABA immunoreactivity, but are surrounded by numerous GABA-positive terminals $(B)$. $C, D$, Cat-301 staining $(C)$ of layer $\mathrm{V}$ cells, 2 of which are large pyramidal cells $(P)$ covered along the surfaces of their somata and dendrites with GABA-positive terminals $(D)$. A third, smaller cell (arrow) is both Cat-301- and GABA-positive. Bar, $30 \mu \mathrm{m}(A-D) . E, F$, Cat-301 $(E)$ and GABA $(F)$ staining of 2 neurons (arrows) in layer IIIB. These multipolar, nonpyramidal neurons resemble basket cells in being the largest of the GABA-positive neurons in area 4. Bar, $15 \mu \mathrm{m}$.

layers IVB and VI contain a large number of Cat-301-positive neurons (Figs. $6 E, 7 B$ ). At the border with area 18 , the 2 bands of intensely stained cells in area 17 give way to more lightly stained bands in layers IIIB and V (Fig. $7 B$ ). The change clearly marks the border between these areas. Similar, though less dramatic, changes in Cat-301 staining are evident at the anterior and posterior borders of area 3 in the first somatic sensory cortex (Fig. $8 A$ ). The cells in area 3 are relatively lightly stained and are concentrated in layers IV and VI, while those in the more anterior area 4 and the more posterior areas 1-2 are heavily stained and occupy major bands in layers IIIB and $\mathrm{V}$ (Fig. $8 A$ ). Much broader areal differences exist within the cat and mon- 
Figure 5. Correlation of GABA immunoreactivity and Cat-301 staining of neurons in cat area 17. A, Fluorescence photographic montage of Cat-301stained cells through the depth of area 17. Cells are stained in all layers but are densest in 2 bands. $B$, A second montage of the same section, showing GABA-immunoreactive cells in area 17. These neurons are also found in all layers. Arrows in $A$ and $B$ indicate some of the many neurons that are both Cat301-and GABA-positive. Bar, $100 \mu \mathrm{m}$.
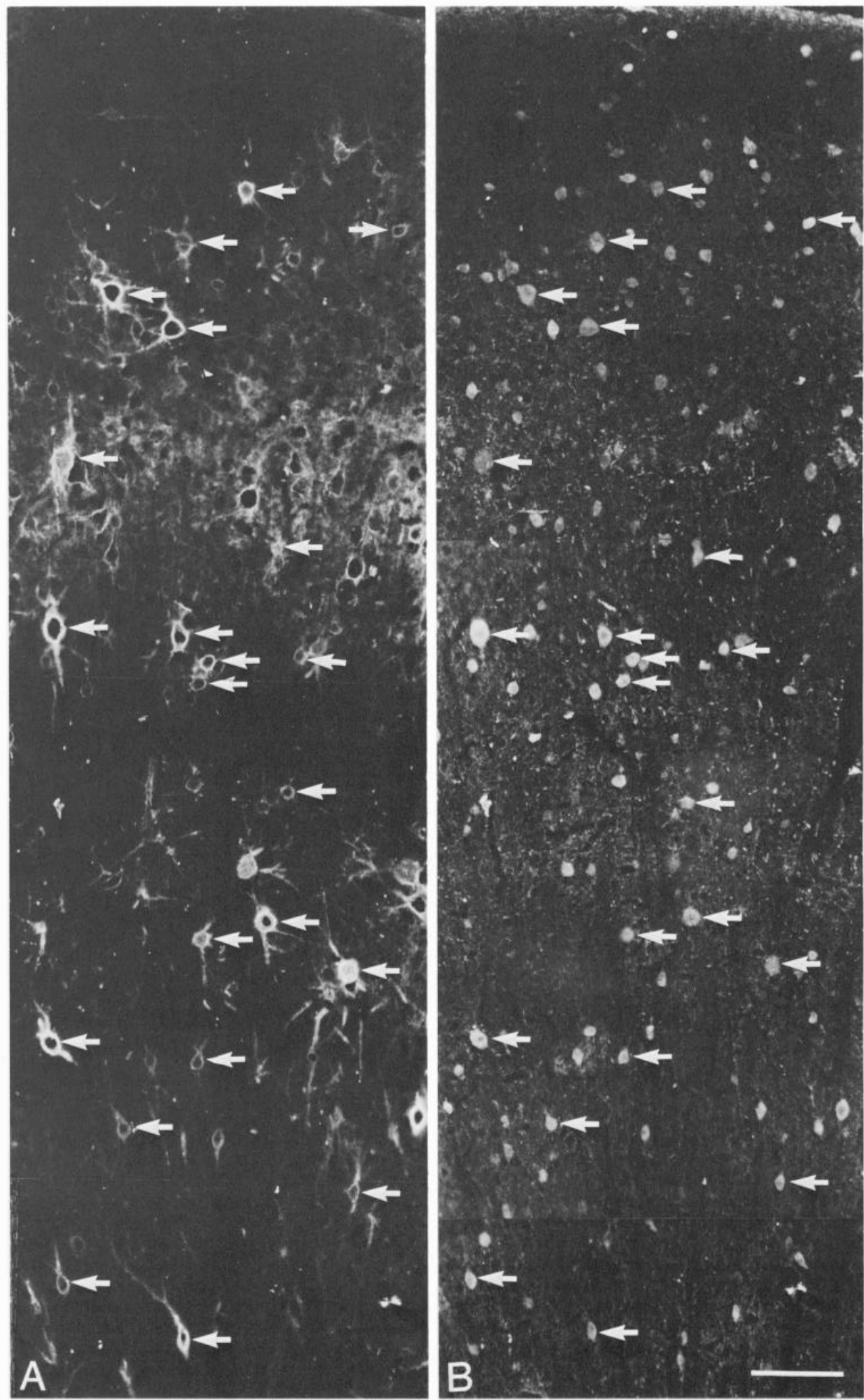

key cortex, based on gross differences in the numbers of Cat301-positive cells. In both species, a wide expanse of cortex that includes the sensory-motor areas, the primary and secondary visual areas (17 and 18), and the associational areas of the suprasylvian gyrus contain the highest densities of immunoreactive cells (Figs. 8, 9). By contrast, a large block of cortex, including the entire temporal region, with the auditory areas and the insular cortex, contains relatively few stained neurons (Figs. 8,9). Areas of the frontal lobe and the areas of the cingulate gyrus are also conspicuous for their very low density of stained cells (Figs. 8, 9).

Species differences. Differences exist among species in the 

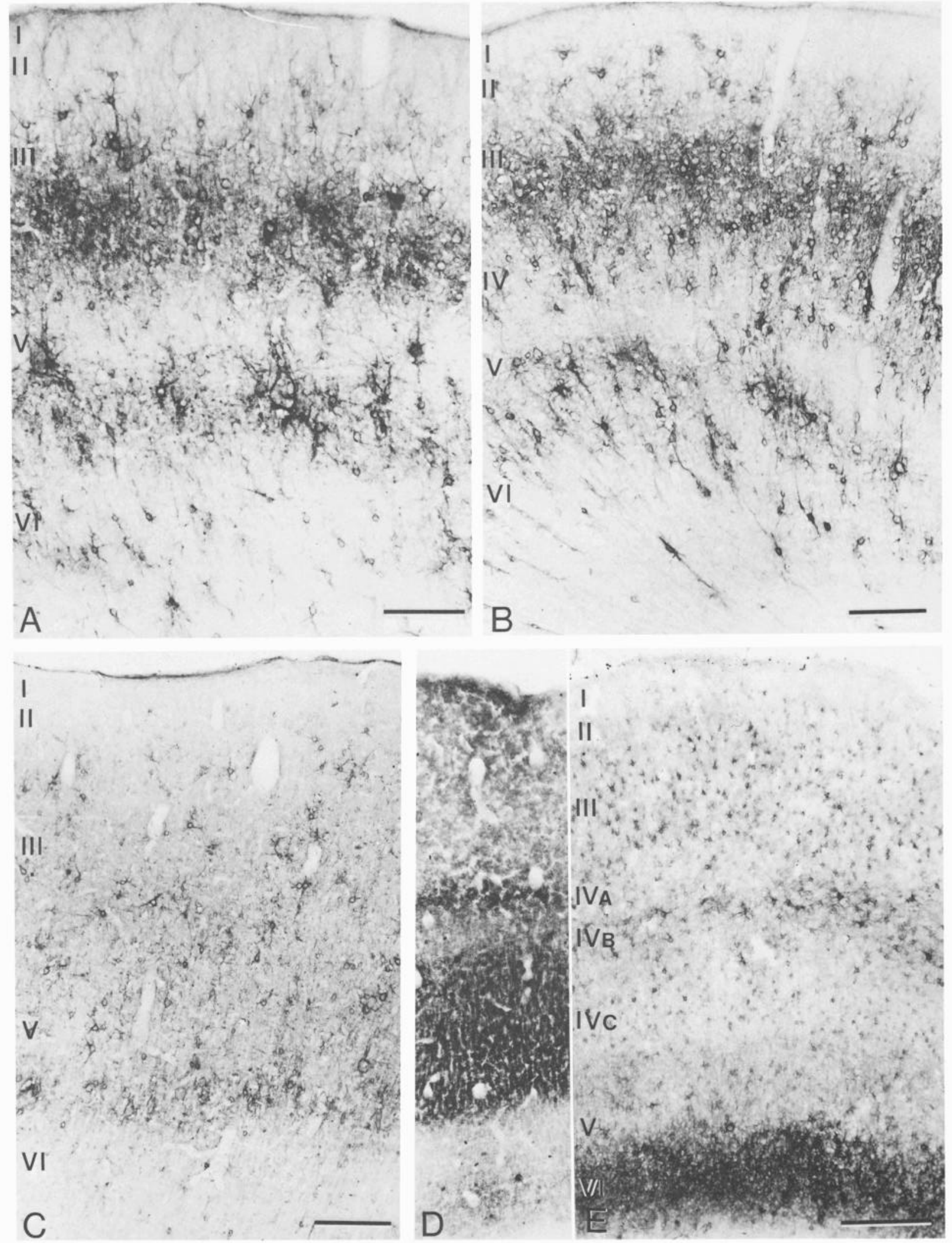

Figure 6. Laminar organization of Cat-301-stained neurons in cat and monkey cerebral cortex. In area 4 of both cats $(A)$ and monkeys $(C)$, numerous Cat-301-positive cells occupy 2 bands, corresponding to the deeper halves of layers III and V. However, the density of cells within these layers is much greater in the cat and, thus, the bands stand out more clearly. Within cat $(B)$ and monkey $(E)$ area 17 , a relatively large number of Cat-301-positive cells are found in layer IV or in subdivisions of that layer, but these species differ in the distribution of cells in the deeper layers: a dense band is present in deep layer V of cat area 17, while the densest staining is found in layer VI of monkey area 17. The photomicrograph in $D$ is of the section adjacent to the one in $E$, but stained histochemically for CO to delineate the laminar boundaries. Bars: $90 \mu \mathrm{m}(A, B) ; 250 \mu \mathrm{m}$ $(C-E)$. 




Figure 7. Areal differences in Cat-301 staining of cat and monkey cerebral cortex. A, Low-magnification photomicrograph of Cat-301 staining in the cat cortex. Dense neuropil staining is found in layers containing numerous Cat-301-positive cells. The patterns of cell-surface and neuropil staining serve to mark the borders (arrows) between areas of cortex, which in this section include areas 17, 18, 19, and 7, and the lateral suprasylvian area. The cingulate gyrus $(C G)$ is lightly stained. The areal boundaries were determined cytoarchitectonically from an adjacent thionin-stained section. Bar, $300 \mu \mathrm{m}$. B, Low-magnification photomicrograph of monkey visual cortex. The densest Cat-301 staining in this area is found in layer VI of area 17 , where numerous immunoreactive somata are embedded in a densely stained neuropil. The intense layer VI staining stops abruptly at the border between areas 17 and 18 (arrow). Bar, $400 \mu \mathrm{m}$.

patterns of Cat-301 staining, even for the same functional areas of cortex. The differences include variations not only in the number of Cat-301-positive cells and in the proportion that displays GABA-like immunoreactivity (see above), but also in the laminar distribution of the cells and the intensity of their staining. These differences can be seen by comparing the staining of areas 4 and 17 in cats and monkeys (Fig. 6). Broader differences are seen with the other species. Many neurons in the guinea pig cerebral cortex are immunoreactive, but the number of cells is still much lower than in cat and monkey cortex and is significantly lower than in several subcortical sites of the guinea pig brain, such as the claustrum. In the rat cortex, only neurons of the hippocampal formation are Cat-301-positive (Fig. 1B); in the bush baby cortex, very few, widely scattered stained cells are present in any part of the cortex.

Columnar organization of Cat-301-positive neurons in monkey visual cortex. We have reported previously (Hockfield et al., 1983; Hendry et al., 1984a) that in the monkey primary visual cortex, the major concentrations of Cat-301-positive neurons are found in layers IVB and VI. In layer IVB, most Cat-301positive neurons are large ( $15 \mu \mathrm{m}$ in diameter), closely packed nonpyramidal cells with long, intensely stained processes. Near the nonpyramidal cells are a few, very large Cat-301-positive cells with triangular somata ( $>20 \mu \mathrm{m}$ in diameter) that give off single prominent ascending dendrites and are probably pyramidal cells. In layer VI, a large, mixed population of neurons and the surrounding neuropil are stained. The stained neurons include many with small somata and others with large (15-20 $\mu \mathrm{m}$ in diameter) triangular somata. Cat-301-positive cells are also found in other layers of monkey area 17 (Figs. $6 E, 7 B$ ), including layers II-IVA, IVC, and V, where small neurons (somata $8-12 \mu \mathrm{m}$ in diameter) give rise to one or 2 short, stained processes.

The paired photomicrographs in Figure 10 are from adjacent sections cut parallel to the pial surface through area 17 of a normal monkey. The section in Figure 10, left, was stained with Cat-301 and that in Figure 10, right, was stained for CO. Both sets of photomicrographs show that the staining in layers II-III is patchy. The patches stained for $\mathrm{CO}$ in layers II-III average $120 \times 180 \mu \mathrm{m}$ in diameter and, in sections cut parallel to the pial surface, line up in rows with a center-to-center spacing of $450 \mu \mathrm{m}$ (Fig. 10B). Those stained with Cat-301 are smaller, averaging $90 \times 140 \mu \mathrm{m}$, but also line up in rows, with a spacing of $450 \mu \mathrm{m}$ (Fig. 10A). By superimposing the profiles of blood vessels that run radially through the thickness of the cortex, we compared the distribution of $\mathrm{CO}$ and Cat-301 patches. As seen in Figure 10, the patches of Cat-301-positive cells deep in layer III consistently coincide with the CO patches. In layer II and the superficial part of layer III, the Cat-301-positive cells are more evenly dispersed, and form clusters that are not as distinct as those more deeply situated. The relationship between the $\mathrm{CO}$ and Cat-301 staining in layer II and superficial layer III is not as consistent as that in deep layer III. In some places, the 2 appear to be complementary, with the Cat-301-positive cells occupying zones between the CO-stained patches. Still, in many other places, the clusters of stained cells and the patches of intense $\mathrm{CO}$ staining coincide precisely, as they do in deep layer III (Fig. 10, $A, B$ ).

Although the patches of Cat-301-positive cells deep in layer III coincide with the patches of intense $\mathrm{CO}$ staining, the 2 are not entirely coextensive. First, $20 \%$ of the CO patches have no corresponding patch of Cat-301-stained cells. These $\mathrm{CO}$ patches without Cat-301-positive equivalents adopt no obvious pattern and appear to be randomly distributed in the horizontal dimension. Second, most patches of Cat-301-positive cells are smaller than their CO-stained counterparts. Comparison of adjacent sections reveals that the Cat-301-stained patches occupy the central, most darkly stained cores of the $\mathrm{CO}$ patches and, 

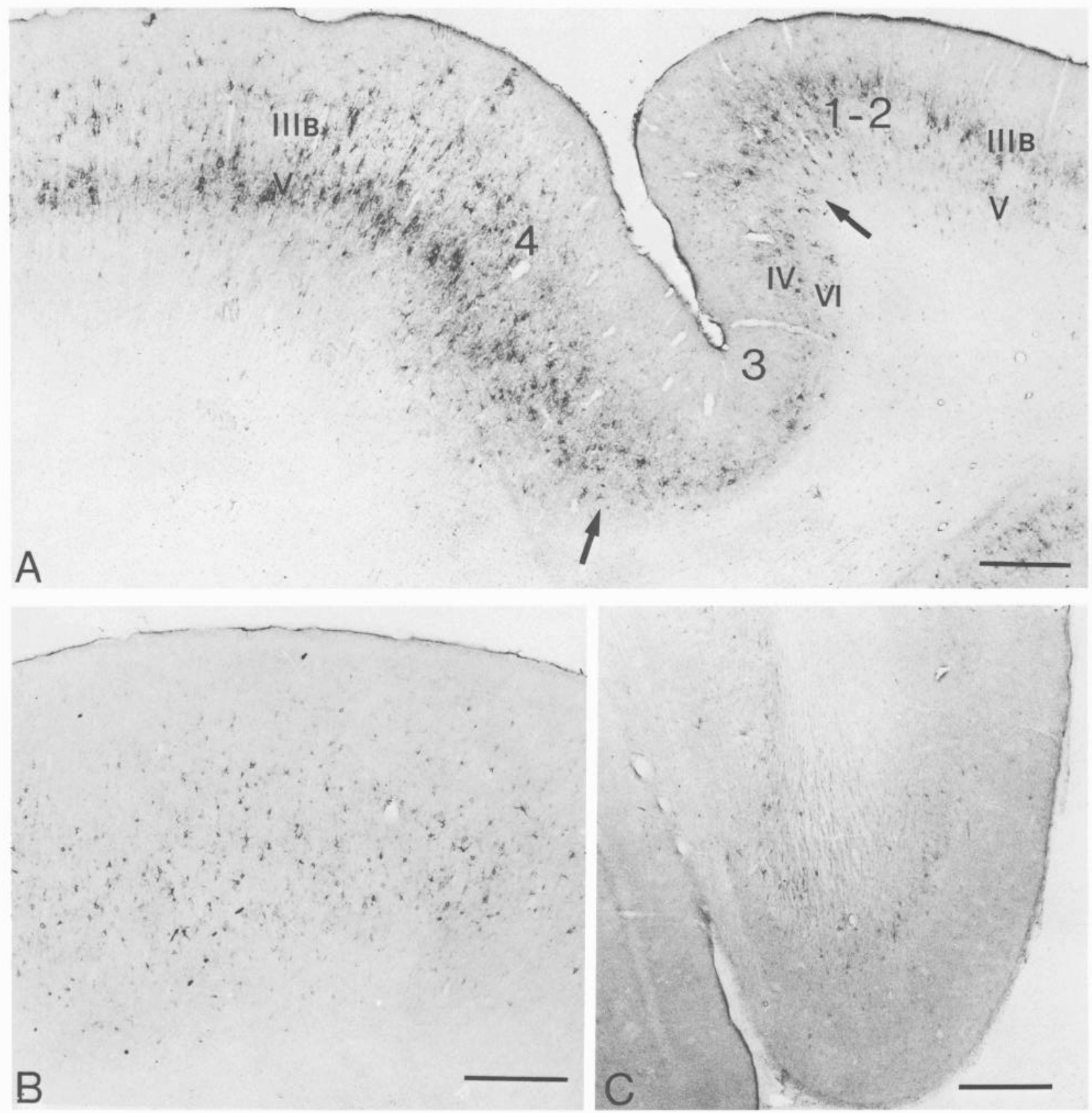

Figure 8. Distribution of Cat-301-positive cells in the monkey cortex. A, Photomicrograph of a sagittal section through the central sulcus, including the precentral motor area (area 4) and the first somatic sensory cortex (areas 3 and 1-2). Dense bands of stained cells in areas 4 and 1-2 include layers IIIB and V, while in area 3 the staining is much lighter and most concentrated in layers IV and VI. Arrows indicate approximate positions of cytoarchitectonic borders. B, Frontal section through the dorsal prefrontal cortex. As compared with the sensory-motor areas, fewer Cat-301positive cells are present and these are more evenly distributed across layers. $C$, Frontal section through the temporal lobe, showing the presence of Cat-301-positive cells in the presubiculum. There are few stained neurons in the monkey temporal cortex. Bars, $750 \mu \mathrm{m}$.

thus, occupy only one-half to two-thirds of the latter (Fig. 10, $C, D)$.

As shown in Figure 11, the patches of Cat-301-positive cells are present in layers IVB and VI of area 17, and these also line up in rows that are perpendicular to the area $17 / 18$ border (Hockfield et al., 1983; Hendry et al., 1984a). Figure 11 shows the distribution of Cat-301-positive cells in area 17 of a monkey from which one eye had been removed $7 \mathrm{~d}$ before it was killed. Unlike the normal, uniform CO staining in layer IVC, the staining in an enucleated monkey consists of alternating darkly and lightly stained strips, $400-600 \mu \mathrm{m}$ wide (Fig. $11 B$ ). These correspond to ocular dominance columns associated with the intact eye and removed eye, respectively (Horton and Hubel, 1981). The photomicrograph in Figure $11 A$ shows that the Cat-301positive cells in layer IVB, overlying the CO-stained bands in IVC, are grouped into patches that line up in parallel rows. When profiles of the same radially oriented blood vessels are aligned in the 2 micrographs, the rows of Cat-301 patches can be lined up with the centers of both the darkly and lightly stained $\mathrm{CO}$ strips. 

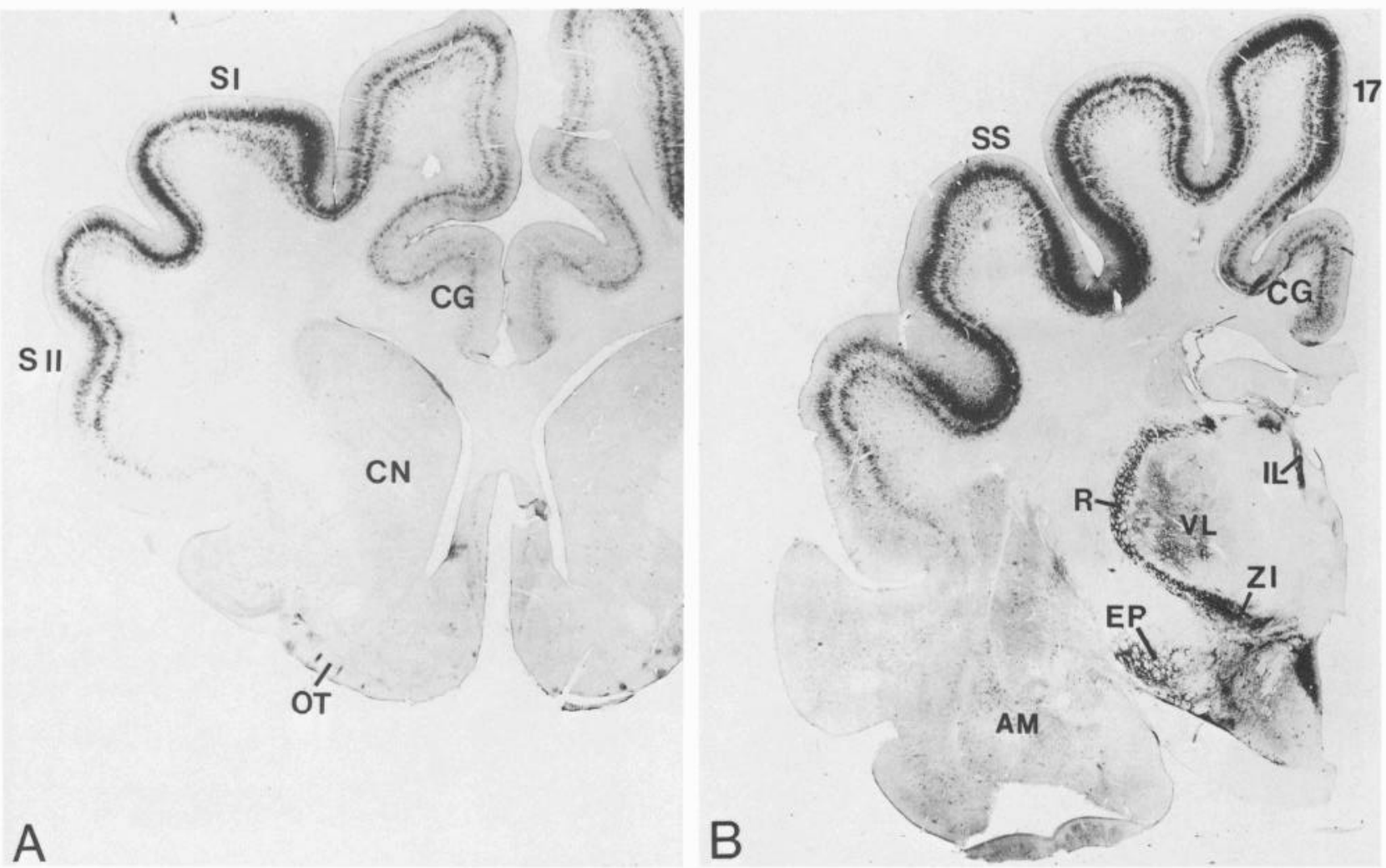

A

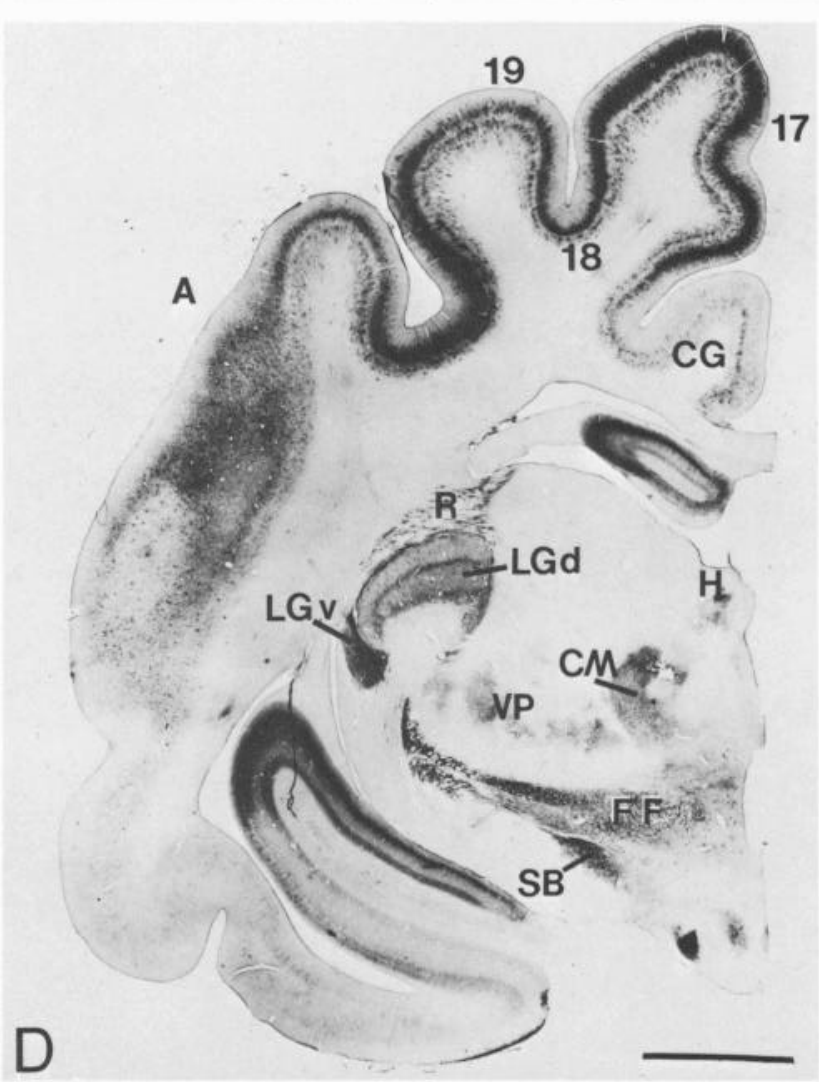

Figure 9. Cat-301 staining of the cat forebrain. $A-D$, Photomicrographs of a series of frontal sections through the cerebral hemisphere and diencephalon. In the cortex, intense staining is seen in the first somatic sensory area $(S I)$, the primary visual area (area 17), and in the middle suprasylvian gyrus $(S S)$. Less intense staining is found in the auditory $(A)$, cingulate $(C G)$, and second somatic sensory $(S I I)$ areas. Very few Cat301-positive cells are present in the pyriform lobule. The patches in the olfactory tubercle $(O T)$ are regions of intense cell and neuropil staining within the islands of Calleja. Scattered CAT-301-positive cells are present in the amygdala $(A M)$ and caudate nucleus $(C N)$. In the diencephalon, intense staining is found in the reticular $(R)$, anterodorsal $(A D)$, intralaminar $(I L)$, and ventral thalamic nuclei $(V L$ and $V P)$, and in both the dorsal and ventral lateral geniculate nuclei $(L G d$ and $L G v)$. Cell and neuropil staining are also present in the zona incerta $(Z I)$, the subthalamic nucleus $(S B)$, the field of Forel $(F F)$, and the entopeduncular nucleus $(E N)$. Within the hypothalamus, the paraventricular nucleus and the mamillary complex are stained, and in the epithalamus, the habenular complex $(H)$ contains Cat-301-positive cells. Bar, $5 \mathrm{~mm}$. 



Figure 10. Relationship of Cat-301 patches and CO patches in the supragranular layers (I-III) of normal monkey visual cortex (area 17). A, Tangential section through area 17 cut parallel to the opercular surface of the occipital lobe and stained with Cat-301. The stained cells deep in layer III (below the lines) form patches that line up in rows. Cells in superficial layer III and in layer II are more evenly dispersed and form fewer, less distinct clusters. $B$, Section adjacent to $A$, stained for $C O$. Densely stained patches form rows in layers II and III. Comparison of the positions of the same blood vessels (arrows) in $A$ and $B$ shows that, in deep layer III, the patches of Cat-301-positive cells coincide with the dark CO patches. At more superficial levels, the relationship between the $\mathrm{CO}$ patches and the Cat-301-positive cells is less consistent; many clusters of stained cells coincide with the $\mathrm{CO}$ patches, while other stained cells are present in the zones between the patches. $C, D$, Higher-magnification photomicrographs of $A$ and $B$, showing patches of Cat-301-positive cells and dark CO staining in deep layer III. Comparison of these micrographs show that the patches of stained cells occupy the central, most darkly stained cores of the CO patches. Bars, $500 \mu \mathrm{m}$. 


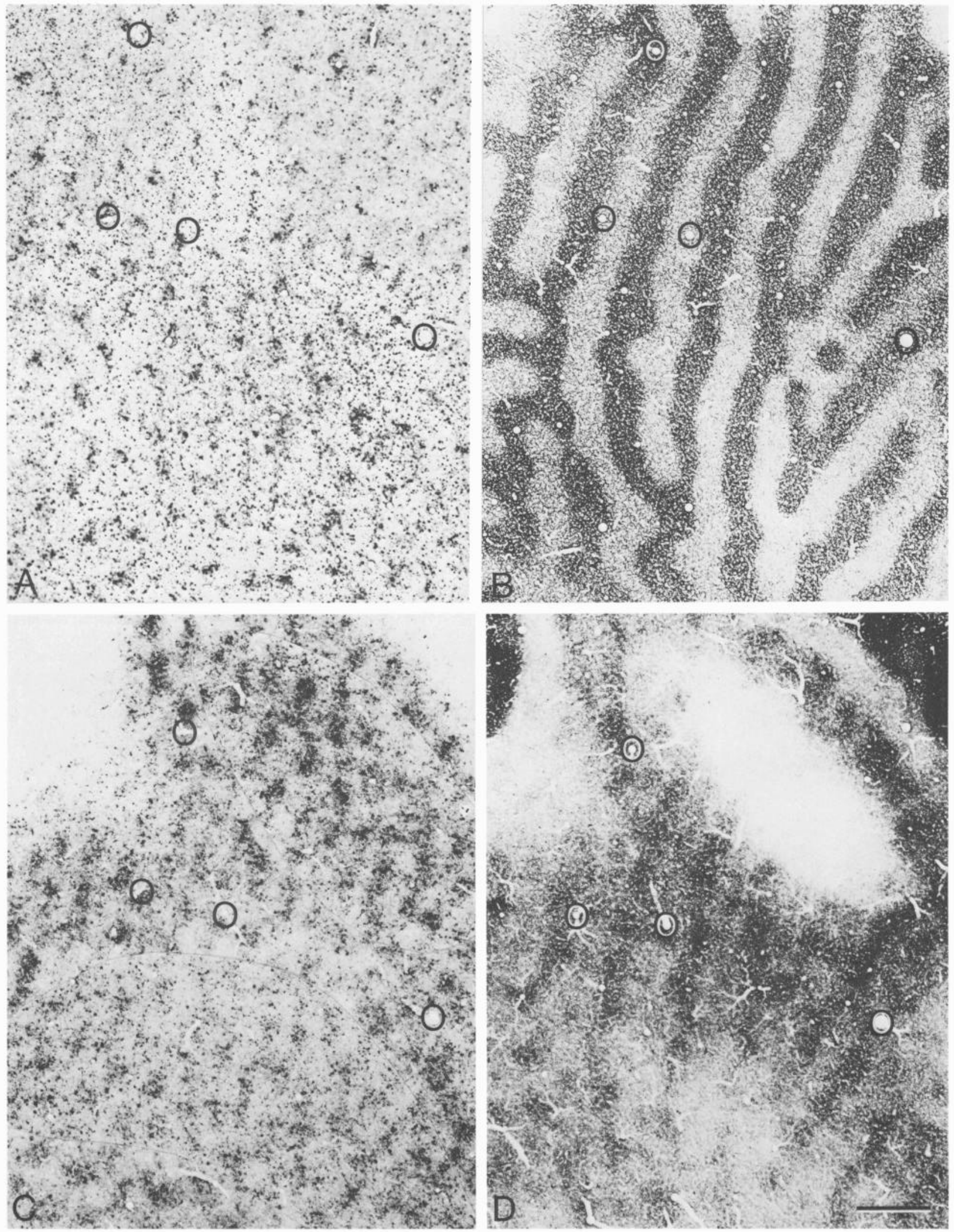

Figure 11. Relationship of Cat-301 patches to ocular dominance columns in area 17 of a monocularly enucleated monkey. $A, C$, Cat-301 staining in layers IVB $(A)$ and VI $(C)$ consists of patches of cells, 100-125 $\mu \mathrm{m}$ in diameter. The patches are more darkly stained in layer VI, but in both layers they form rows. Comparison of the positions of the same blood vessels (circles) in the 2 sections reveals that the patches in layer IVB line up radially with those in layer VI. Only the central two-thirds of $A$ include the relatively thin layer IVB. The upper part of the photomicrograph includes layer IVA and the lower left edge, layer IVC, neither of which contain patches of cells. $B, \mathrm{CO}$ staining in layer IVC, $120 \mu \mathrm{m}$ deep to the section in $A$. Alternating light and dark CO bands, $350-500 \mu \mathrm{m}$ wide, correspond to ocular dominance columns for the removed and intact eyes, 
The association between the Cat-301 patches and the system of ocular dominance columns is also apparent in layer VI. In unilaterally enucleated monkeys, the rows of $\mathrm{CO}$ patches in this layer are not equally stained. Instead, patches in every other row, associated with the removed eye, are pale and shrunken, while those in intervening rows, associated with the intact eye, are relatively large and intensely stained, appearing as thin stripes (Fig. 11D). The Cat-301 staining in layer VI of both intact and enucleated monkeys is made up of patches of neurons that line up in parallel rows (Fig. 11C), but in the enucleated animals the neuropil staining between the rows decreases in intensity, making the patchy nature of the staining more distinct. Alignment of profiles of the same blood vessels in Figure $11, C, D$ demonstrates that the rows of Cat-301-stained patches coincide with both the darkly stained and lightly stained rows of $\mathrm{CO}$ patches, confirming that the Cat-301 patches lie at the centers of both sets of ocular dominance columns.

The clusters of Cat-301-positive cells in area 17 are made up principally of GABA-immunoreactive neurons. The patches of small Cat-301-positive cells in layers II-III (Fig. 12, $A, B$ ) and the patches of larger stained cells in layer IVB (Fig. 12, $C, D$ ) contain Cat-301-positive neurons that are also immunostained for GABA. The double-labeled cells are a fraction of the entire, more evenly dispersed, population of GABA neurons in these layers. The Cat-301-positive cells in layer VI of area 17 also include small, GABA-immunostained neurons and larger neurons that display no GABA immunoreactivity (Fig. $12, E, F$ ).

The photomicrographs in Figure 13 show that the patterns of $\mathrm{CO}$ and Cat-301 staining are also nonhomogeneous in area 18. The intense $\mathrm{CO}$ staining in area 18 consists of a series of bands varying in width from 200 to $300 \mu \mathrm{m}$ to as much as a millimeter (Livingstone and Hubel, 1982; Fig. 13A). The bands are continuous through layers IIIB-VB. In sections stained with Cat301 , bands of immunoreactive cells are also evident in layers IIIB and VB (Fig. 13A). The bands are apparent because of the larger number of Cat-301-positive cells within them, as compared with the regions between them. The bands of greater Cat301 staining coincide with every other band of intense CO staining (Fig. 13). For the most part, the Cat-301 staining appears to occupy an area equal to that of the $\mathrm{CO}$ staining, although, in some cases, the stained neurons occupy a thinner region that includes only the central two-thirds to three-quarters of a $\mathrm{CO}$ stained band.

Other areas of cat and monkey cortex were examined for periodic Cat-301 staining. In some areas, such as the sensorymotor areas of the monkey and area 17 of the cat, the Cat-301positive cells are distributed nonhomogeneously, with bands or patches of cells being separated by more lightly stained regions. However, in no area do the Cat-301-positive cells adopt a simple, regular pattern in the horizontal dimension, as was seen in areas 17 and 18 of the monkey.

\section{Cat-301 staining of the thalamus}

Cell types. Within the nuclei of the cat and monkey thalamus, only large neurons (18-26 $\mu \mathrm{m}$ in diameter) are Cat-301-positive
(Fig. 14). In some thalamic nuclei of both species [e.g., the ventral posterior lateral (VPL) and ventral posterior medial (VPM)] the stained neurons give off 4 or 5 stained processes, the largest of which divide repeatedly close to their parent somata and give rise to a tuft of finer stained branches (Fig. 14A). In other nuclei, such as the dorsal lateral geniculate nucleus (LGN), the Cat-301-positive neurons do not have extensively stained processes.

The neurons of the dorsal thalamus stained by Cat-301 are exclusively relay neurons projecting to the cerebral cortex. Figure $14, D, E$ shows that neurons in VPL labeled by the retrograde transport of fast blue from the ipsilateral SI (Fig. 14E) are Cat301 -positive (Fig. 14D). In contrast with findings in the cerebral cortex, no GABA-immunoreactive neurons in the monkey thalamus, except for those in the reticular nucleus (see below), are stained with Cat-301 (Fig. 14, B, C). Instead, the largc Cat-301positive cells in the dorsal thalamus are covered along the stained surfaces of their somata and dendrites with numerous GABApositive terminals (Fig. 14, $B, C$ ).

Cat-301 staining across thalamic nuclei. Like Cat-301-positive cortical neurons, the thalamic cells are nonhomogeneously distributed, with the density and distribution of stained neurons differing markedly among nuclei and species. In the following description, the nuclei are named according to Jones (1985).

In the dorsal thalamus of cats and monkeys, members of a restricted number of nuclear groupings are stained (Figs. 9, 15). These include the anterodorsal nucleus in the anterior group, the rostral nuclei (central lateral, central medial, and paracentral) of the intralaminar group, and the suprageniculate-limitans and posterior nuclei of the posterior group. In the ventral group, VPL, VPM, and the ventral lateral anterior (VLa) and ventral lateral posterior (VLp), nuclei contain dense collections of Cat301-positive cells (Fig. 15, $A-D$ ). Stained cells are also found throughout the LGN, although they are not evenly stained or distributed through this nucleus (Fig. 15, $C, D$; see below). In the medial geniculate complex, stained cells are restricted to the magnocellular nucleus. With the exception of solitary, intensely stained cells that are found in some nuclei, particularly the lateral posterior nucleus and the anterior pulvinar nucleus, all other dorsal thalamic nuclei are unstained.

The ventral thalamus of the monkey is made up of the reticular and pregeniculate nuclei and the nuclei of the zona incerta and of the field of Forel. All contain neurons and neuropil stained with Cat-301 (Fig. 15). The staining divides the pregeniculate nucleus into a darkly stained dorsal cap and a lightly stained ventral region (not illustrated). Within the reticular nucleus, virtually all neurons are stained, and these are distributed in thin, finger-like clusters (Fig. 15). All of the Cat-301-positive cells in the reticular nucleus are also immunoreactive for GABA (not illustrated). In the cat ventral thalamus, the ventral LGN is heavily stained, with large immunoreactive cells tending to form clusters in more dorsal regions of the nucleus (Fig. 16B).

Intranuclear organization of Cat-301-positive neurons. The distribution of Cat-301-positive cells in the LGN of monkeys and cats and in the VPL and VPM of monkeys is not uniform.

respectively. The CO bands have the same orientation as the rows of Cat-301-stained patches in layer IVB $(A)$, and when the positions of the same blood vessels (circles) are compared in $A$ and $B$, the Cat-301-positive rows in layer IVB are found to line up with the centers of both sets of ocular dominance columns. $D$, CO staining in layer VI is made up of rows of patches. In every other row, corresponding to columns dominated by the intact eye, the patches are larger and more darkly stained, forming thin, continuous stripes. In the alternating rows, the patches are shrunken and pale. By comparing the positions of the same blood vessels (circles) in $C$ and $D$, it can be seen that the Cat-301-stained rows line up with both the lightly and darkly stained $\mathrm{CO}$ rows. Bar, $1 \mathrm{~mm}$. 

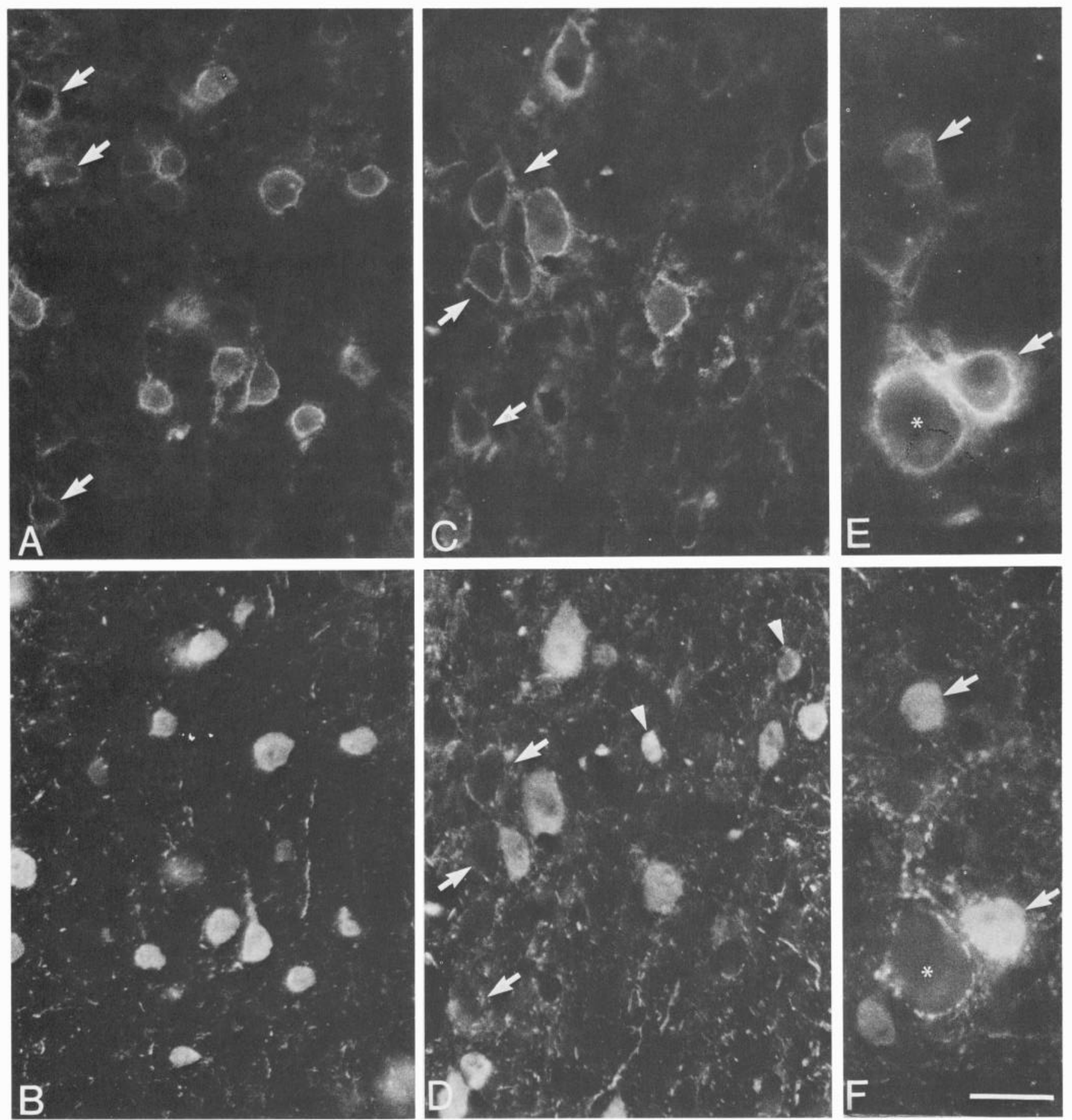

Figure 12. Cat-301 and GABA staining of patches of neurons in monkey area 17. A, B, Cat-301 $(A)$ and GABA $(B)$ staining of a patch of neurons deep in layer III. All of the Cat-301-positive cells within the patch are small and most are also GABA-immunoreactive. However, some of the Cat-301-positive cells (arrows) display no GABA immunoreactivity. $C, D$, Cat-301 $(C)$ and GABA $(D)$ staining of a patch of neurons in layer IVB. Most of the Cat-301-positive cells are relatively large and also GABA-positive, but some large cells (arrows) display no GABA immunoreactivity and are surrounded only by GABA-positive terminals. A few small GABA-positive cells (arrowheads) are not stained by Cat-301. $E, F$, Cat-301 $(E)$ and GABA $(F)$ staining in part of a layer VI patch. Of the 3 Cat-301-positive cells that are shown, the 2 smaller cells (arrows) are also GABApositive, while the largest $\left(^{*}\right)$ is covered with GABA-positive terminals but is not, itself, GABA-positive. Bar: $25 \mu \mathrm{m}(A-D)$; $15 \mu \mathrm{m}(E, F)$.

As we have reported earlier (Hockfield et al., 1983; Hendry et al., 1984a), 2 prominent bands of intense Cat-301 staining are present in the monkey LGN, corresponding to the magnocellular layers (Fig. 16A). Counts indicate that approximately $70 \%$ of the neurons in these layers are Cat-301-positive. The 4 dorsal, parvocellular layers appear, by contrast, as lightly stained lam- inae, owing to the lower density of immunoreactive neurons (less than $40 \%$ of the total neuronal population) and the lighter staining of individual cells (Fig. 16A). No Cat-301-positive cells are found either in the $\mathrm{S}$ layer or in the interlaminar zones.

In the cat LGN, many neurons in the magnocellular C lamina, in the medial interlaminar nucleus (MIN), and in the interlam- 

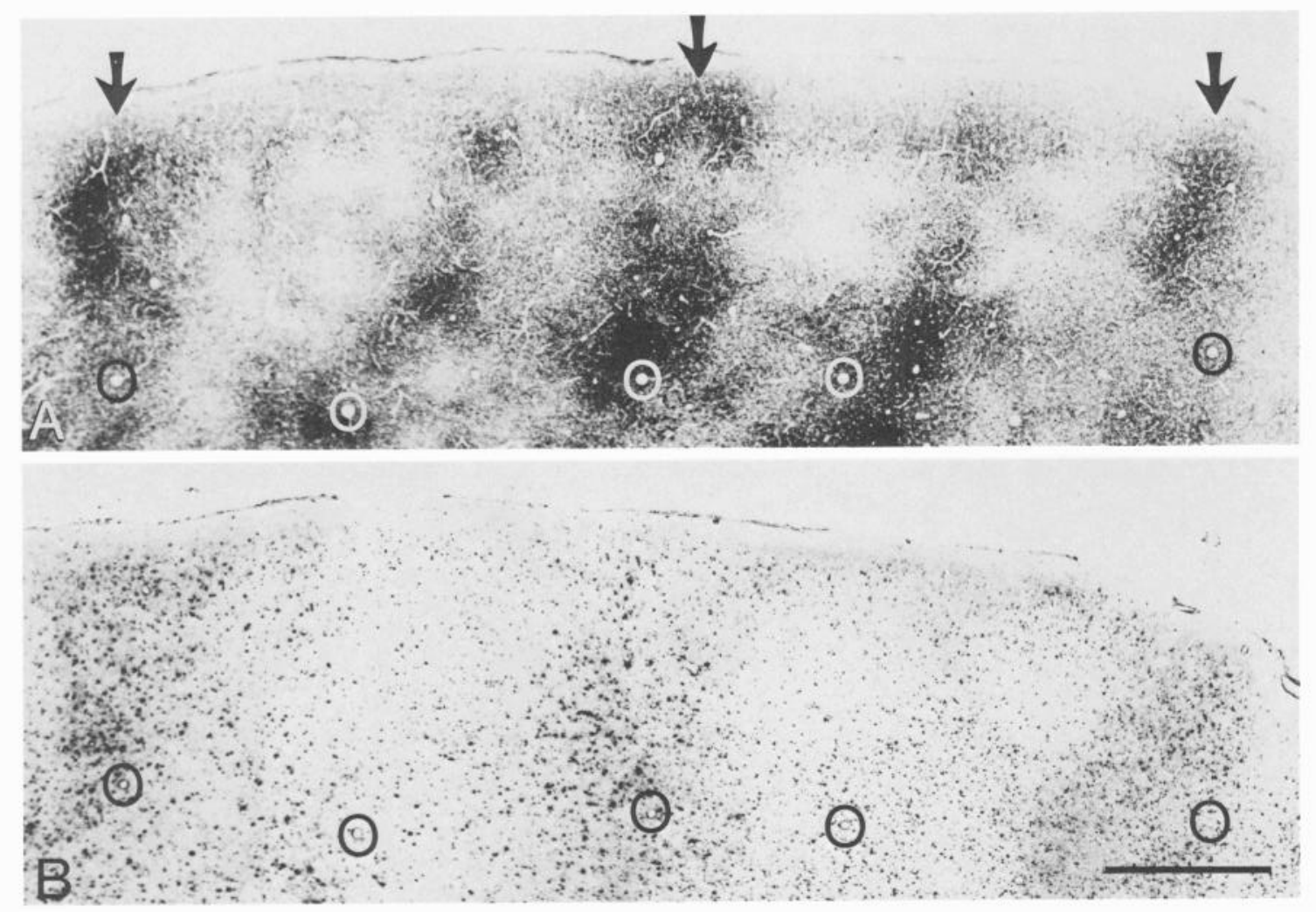

Figure 13. Comparison of Cat-301 and CO staining in monkey area 18. A, Tangential section through the posterior part of the occipital lobe, showing the presence of elongated bands of $\mathrm{CO}$ staining. The bands, oriented perpendicular to the border with area 17 , vary in width from 300 $\mu \mathrm{m}$ to $1 \mathrm{~mm}$. Alignment of the same blood vessels (circles) in $A$ and $B$ shows that every other band of intense CO staining (arrows) corresponds to a band of Cat-301-stained cells in the adjacent section. $B$, Section adjacent to $A$ showing the distribution of Cat-301-positive cells in area 18 . Dense collections of large immunoreactive neurons in layers III-V form bands that coincide with CO-stained bands. Bar, $1 \mathrm{~mm}$.

inar plexuses are Cat-301-positive (Fig. 16B). Laminae A and Al also contain Cat-301-positive neurons, but these are fewer and more lightly stained than in the neighboring regions. Large neurons are stained in $\mathrm{A}$ and $\mathrm{A} 1$, and while they are found throughout both layers, they are concentrated ventrally, adjacent to the interlaminar plexuses. In counterstained sections, the Cat301-positive cells make up a small fraction of the total neuronal population in these layers. Very few stained cells are present in laminae $\mathrm{C} 1-\mathrm{C} 3$, but a large number of intensely stained neurons occupy the entire MIN (Fig. 16B). Small, lightly stained neurons also occupy the extreme lateral margin of the adjacent pulvinar nucleus, corresponding to the retinal recipient zone of the nucleus (Berman and Jones, 1977) or the "geniculate wing" (Guillery et al., 1980).

In VPL and VPM of the monkey thalamus, clusters of 12-30 Cat-301-positive neurons, lying within a densely stained neuropil, occupy areas $200-500 \mu$ m wide (Fig. 16C). The clusters of cells are separated from one another by unstained regions that in some cases approach the size of an average cluster, but most commonly are narrow wedges, no more than $100 \mu \mathrm{m}$ wide. In serial sections, the stained cell clusters line up as rods, elongated in the anteroposterior dimension. Comparable rods have been described recently as termination zones of afferent axons of the medial and trigeminal lemnisci (Jones et al., 1986). The relationship between the afferent rods and the aggregates of Cat301-positive cells was examined by comparing the distributions of trigeminothalamic axon terminations and immunocytochemically stained neurons. The paired photomicrographs in Figure $16, C, D$ show adjacent sections through VPM, one demon- strating anterograde transport of HRP from the contralateral principal sensory nucleus of the trigeminal complex (Fig. 16D), and the other showing the distribution of Cat-301-positive neurons (Fig. 16C). In both sections, patches of staining are separated by regions devoid of staining. Comparison of the micrographs by alignment of blood vessels shows that the staining patterns occupy the same general region but interdigitate with one another. These data indicate that the Cat-301-positive cells lie within the regions of principal sensory trigeminal afferent terminations, but tend to occupy the spaces between the afferent rods.

Differences among species. With the exception of the intranuclear organization in the LGN and VPM, the Cat-301 staining of cat dorsal thalamus is very similar to that of the monkey. This is not the case of the other species we examined. In both the rat and guinea pig, Cat-301-positive cells have a far more restricted thalamic distribution (not illustrated). The intralaminar nuclei in both species contain immunoreactive cell bodies, and these form the sole population of stained thalamic neurons in the rat. In the guinea pig, very dense collections of intensely stained neurons are also found in the reticular nucleus and the zona incerta. Scattered Cat-301-positive cells are also present in the anterodorsal nucleus of this species. Very few Cat-301positive cells are present in the thalamus of the bush baby.

\section{Discussion}

In the mammalian CNS, the search for novel neural antigens has centered on monoclonal antibodies (mABs) specific for single morphological or functional classes of neurons; however, 


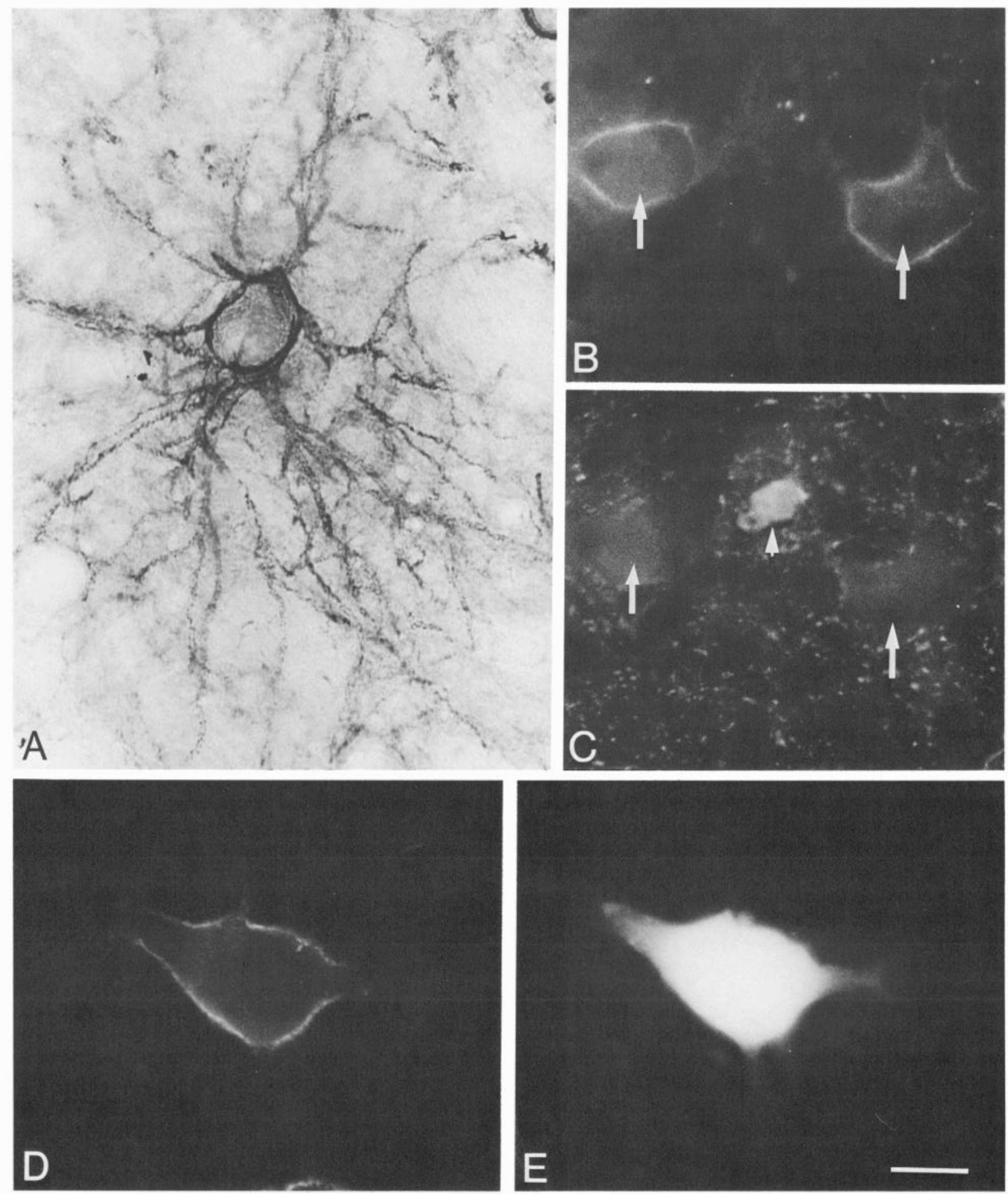

Figure 14. Cat-301 staining of neurons in the monkey thalamus. $A$, Staining of a neuron in the VPL nucleus. In addition to staining of the soma and proximal dendrites, numerous finer branches are stained to give the bushy appearance of a typical relay cell. $B, C$, Cat-301 $(B)$ and $\mathrm{GABA}(C)$ staining in layer 1 of the monkey dorsal LGN. Two large Cat-301-positive cells (arrows) display no GABA immunoreactivity, but are surrounded by GABA-positive terminals. A small GABA-positive cell (arrowhead in $C$ ) is not stained by Cat-301. $D, E$, Cat-301 staining $(D)$ of a large neuron in the monkey VPL nucleus. The same neuron is also labeled by the retrograde transport of Fast blue $(E)$ from injections into the ipsilateral postcentral gyrus. Bar, $10 \mu \mathrm{m}$.

with the exception of certain regions, notably the retina (Barnstable, 1980; Young and Dowling, 1984; Fry et al., 1985) and the cerebellar cortex (Hawkes et al., 1982, 1985), such specificity has rarely been encountered. In the present study, we focused on the staining of neurons by a single $\mathrm{mAB}$, Cat-301, in an effort to identify characteristics common among the neurons expressing the antigen. It was recognized in earlier studies that Cat-301 is not specific for a single morphological class of neuron in any 


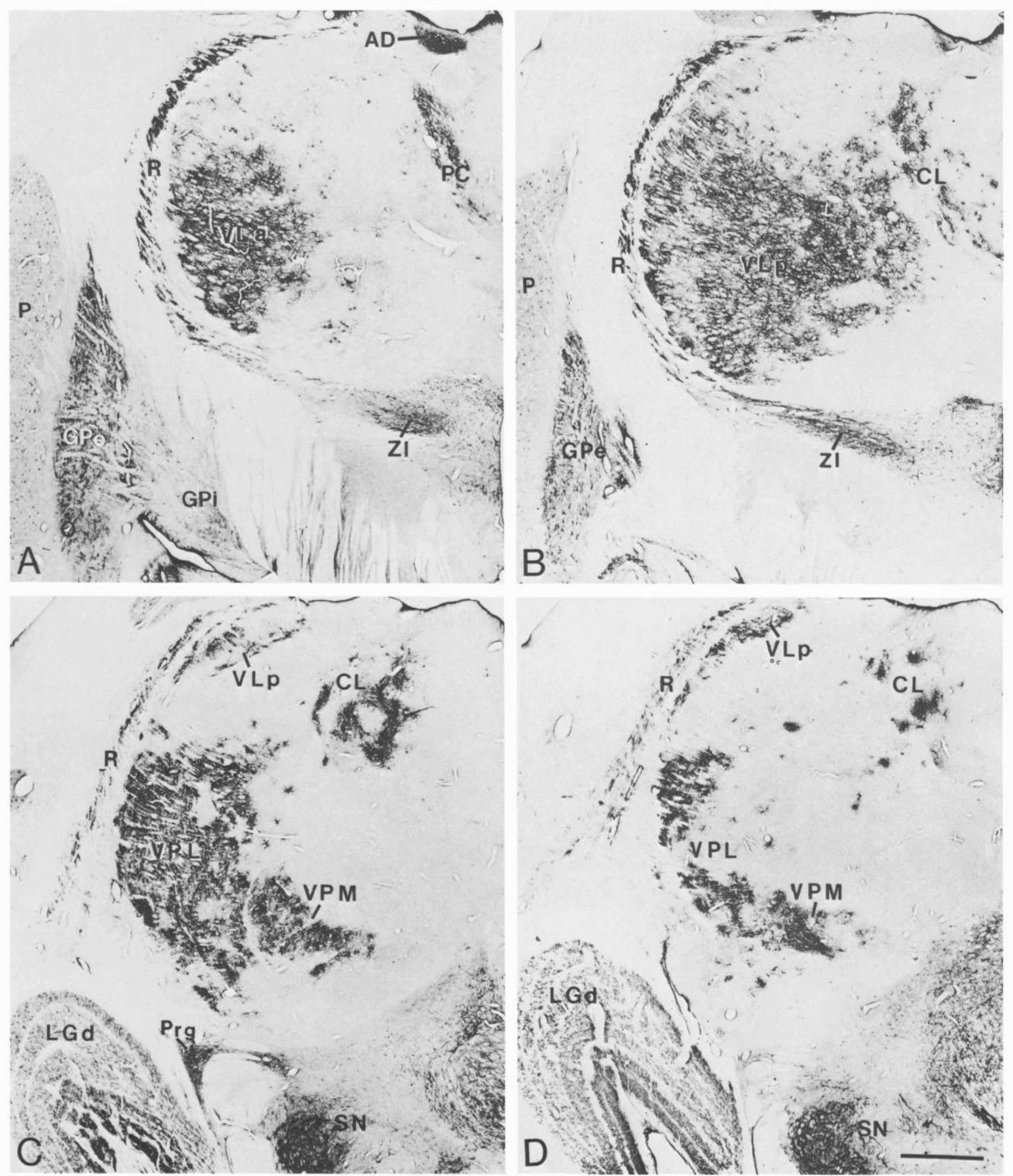

Figure 15. Cat-301 staining of the monkey thalamus and adjacent nuclei. Dense staining of cells and neuropil is present in several nuclear groups, including the anterior group (anterodorsal nucleus; $A D$ ), the intralaminar group (paracentral and central lateral nuclei; $P C$ and $C L$ ), and the ventral group (ventral lateral anterior, $V L a$, and ventral lateral posterior, $V L p$, nuclei, ventral posterior lateral, $V P L$, and ventral posterior medial, $V P M$, nuclei, and the dorsal lateral geniculate nucleus, $L G d$ ). Cat-301-positive cells are also present in the reticular nucleus $(R)$, the pregeniculate nucleus $(\operatorname{Prg})$ and in the zona incerta ( $Z I)$. Some staining is also found in the posterior hypothalamus. Scattered Cat-301-positive cells are present in the putamen $(P)$ and internal segment of the globus pallidus (GPi), while dense staining is found in the external segment of the globus pallidus ( $G P e$ ) and the substantia nigra $(S N)$. Bar, $5 \mathrm{~mm}$. 


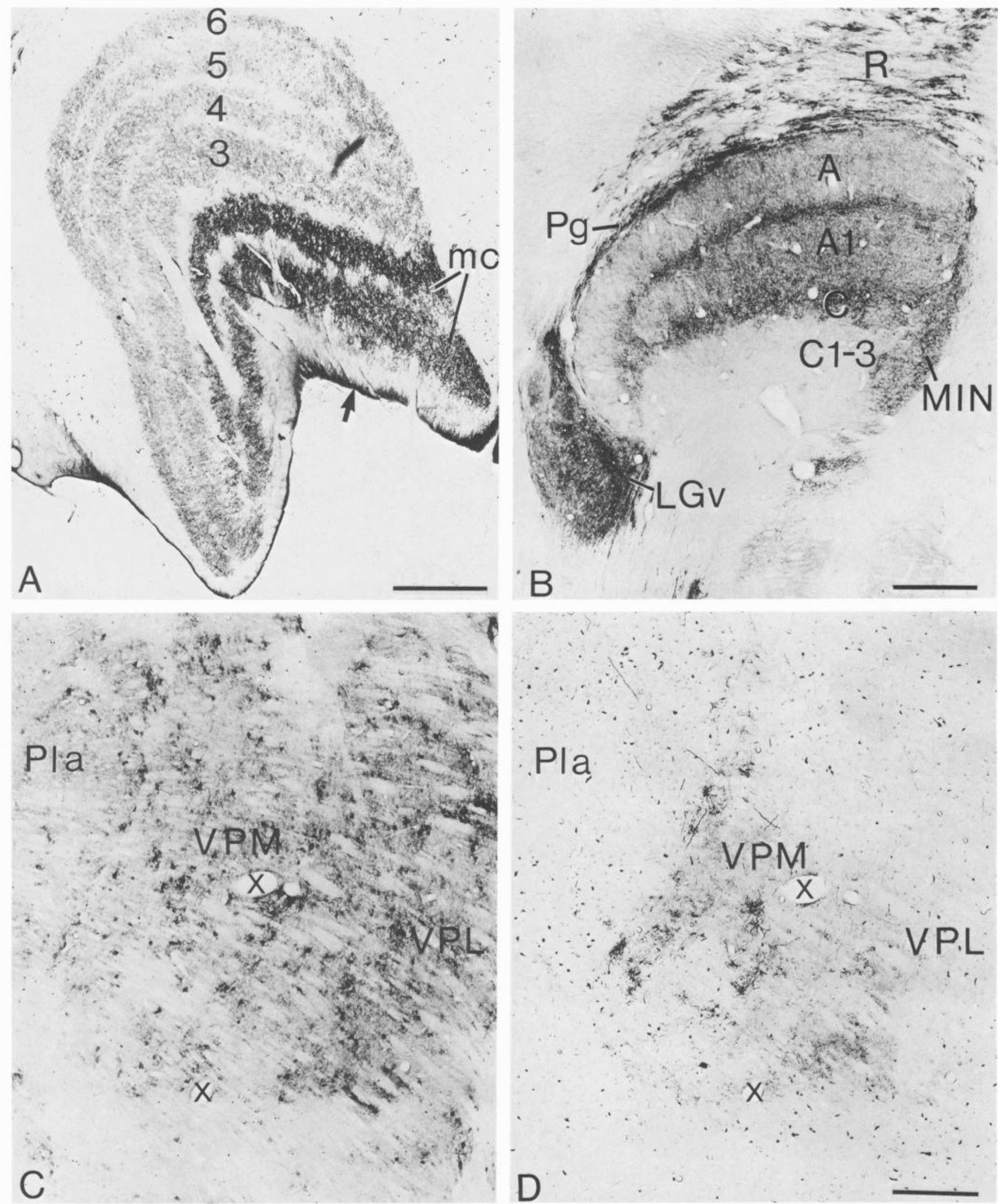

Figure 16. Organization of Cat-301-positive cells in nuclei of cat and monkey thalamus. $A$, Staining with Cat-301 in the dorsal LGN is densest in the ventral magnocellular layers $(m c)$ and includes virtually all of the large projection neurons in those layers. Lighter staining of fewer cells is seen in the dorsal parvocellular layers (3-6). Staining of the pial surface (arrow) is nonspecific. Bar, $200 \mu$ m. B, Organization of Cat-301-positive cells in the cat lateral geniculate complex. In the dorsal LGN, cells in laminae A and Al are stained, as are a few neurons in the ventral C laminae $(C 1-3)$. However, the densest staining is in the interlaminar plexus separating $\mathrm{A}$ and $\mathrm{Al}$, and in lamina $\mathrm{C}$. Numerous Cat-301-positive cells are also present in the medial interlaminar nucleus $(M I N)$, the ventral lateral geniculate nucleus $(L G v)$, the perigeniculate nucleus $(P g)$, and the reticular 
region (McKay and Hockfield, 1982; Hockfield and McKay, 1983a; Hockfield et al., 1983; Hendry et al., 1984a). However, it was also recognized that the $\mathrm{mAB}$ stains groups of neurons that, unlike morphologically definable classes, may correspond to physiologically distinct or anatomically related units (Hockfield et al., 1983; Hendry et al., 1984a). In using Cat-301 to examine more fully the cerebral cortex and thalamus of several mammalian species, we have concluded that Cat-301 stains a diverse collection of morphologically, biochemically, and connectionally distinct neurons that, in some instances, appear to be parts of organized neuronal systems. The present findings will be discussed in relation to 2 broad issues: (1) the organization of the cerebral cortex and thalamus and of neuronal groupings within these regions; (2) the properties that are common among thalamic and cortical neurons expressing the Cat301 antigen.

\section{Cortical and thalamic organization}

Subdivision of pyramidal cell and $G A B A$ cell populations. In the neocortex, Cat-301 is not specific for a single class of cell, but stains both pyramidal and nonpyramidal neurons. These are fundamentally different classes of cells: pyramidal cells include the majority of neurons with axons that leave the cortical area in which their somata lie (Gilbert and Kelly, 1975; Lund et al., 1975; Jones and Wise, 1977), while nonpyramidal cells form the majority of the intrinsic or local-circuit neurons (Valverde, 1971; Jones, 1975; Fairén et al., 1984). It is evident that Cat301 does not stain all members of either the pyramidal or nonpyramidal class, but recognizes subtypes within each class. Differences among pyramidal cells are well known in terms of their connectivity (Lund et al., 1975; Jones and Wise, 1977; White, 1986), the morphology of their axons and dendrites (Gilbert and Wiesel, 1979; Parnavelas et al., 1983; Martin and Whitteridge, 1984), and their functional properties (e.g., Cheney and Fet7., 1980; Evarts et al., 1981), but few differences have been seen in their chemical properties. For example, several lines of evidence suggest that, regardless of the targets of their axons and the laminar position of their cell bodies, pyramidal cells employ the excitatory amino acid glutamate as a neurotransmitter (Streit, 1984). The present study and previous studies employing $\mathrm{mABs}$ (Wood et al., 1982) have detected differences in the immunocytochemical properties of pyramidal cells, including the cells of a single layer. These data suggest that, among the population of pyramidal cells, the expression of certain antigens varies in addition to and possibly in conjunction with differences in connectional, morphological, and functional attributes.

Cat-301 also stains a subpopulation of the GABA-immunoreactive, nonpyramidal cells in both the cat and monkey ccrebral cortex. It is well established that the cortical GABA neurons include several morphologically distinct classes (Houser et al., 1984), each of which appears to occupy a unique position within intracortical circuits by virtue of its synaptic contacts with other pyramidal and nonpyramidal neurons (e.g., Freund et al., 1983; Hendry et al., 1983b; Somogyi and Soltesz, 1986). Chemically distinct subpopulations of GABA neurons have also been identified by their cytoplasmic immunostaining for one or more neuropeptides (Hendry et al., 1984b; Schmechel et al., 1984; Somogyi et al., 1984) and calcium-binding proteins (Celio, 1986), and by their cell-surface staining with other mABs (Arimatsu et al., 1987) or lectins (Nakagawa et al., 1986). Cat-301 does not appear specific for groups of cortical GABA neurons characterized by their morphology or by the expression of other surface or cytoplasmic molecules. The $\mathrm{mAB}$ stains the surfaces of both large and small GABA neurons. The somal size and the largc, predominantly vertical, dendritic field of the largest type suggest it is the large basket cell, for no other cell type conforms to this description (Marin-Padilla, 1969; Jones, 1975; DeFelipe et al., 1986). Among the smaller nonpyramidal cells stained by Cat-301, a variety of morphological types may be present (Jones, 1975; Peters and Regidor, 1981; Fairén et al., 1984). Similarly, a fraction of the Cat-301-positive nonpyramidal cells also display either CCK- or SRIF- and NPY-like immunoreactivities, while the majority exhibit immunoreactivity for no known neuropeptide. These findings indicate that Cat-301 immunostaining defines a unique group of cortical GABA neurons that is heterogeneous in both its morphological and its chemical characteristics.

By contrast with cortical neurons, the neurons of the dorsal thalamus stained by Cat-301 all appear to be of a single broad class - the relay cells that project to the cerebral cortex. Cells labeled by the retrograde transport of fluorescent dyes from the cortex are Cat-301-positive, and none of the GABA-immunoreactive thalamic cells, identified previously as interneurons (Penny et al., 1983; Fitzpatrick et al., 1984; Montero and Zempel, 1985), is stained by the $\mathrm{mAB}$. These findings are similar to those of previous studies in the cat brain stem and spinal cord, which indicate a preferential staining of long-axoned relay cells by Cat-301 (Hockfield and McKay, 1983a). The thalamic relay cells receive synaptic inputs not only from the GABA cells intrinsic to each nucleus, but also from the GABA cells of the thalamic reticular nucleus (Houser et al., 1980; Oertel et al., 1983; Yen et al., 1985). Although the intrinsic GABA cells are not stained with the $\mathrm{mAB}$, all neurons of the reticular nucleus are Cat-301-positive. When the dorsal thalamus and reticular nucleus are considered together, the staining is similar to that of cortical neurons, since in both regions relay cells and a subpopulation of GABA neurons that innervates them express the Cat-301 antigen(s).

Variations among cortical areas and thalamic nuclei. Cat-301 staining varies qualitatively and quantitatively among cortical areas and thalamic nuclei in both cats and monkeys. Broad differences are seen in the cerebral cortex, where some areas contain large numbers of intensely stained cells, while other arcas contain few, widely dispersed, and lightly stained ncurons. These divisions often involve entire lobes, so that areas of the parietal and occipital lobes are intensely stained and most areas of frontal and temporal lobes are lightly stained. The same types of broad differences are seen among groups of thalamic nuclei. In addition, variations in the density and distribution of stained neurons between neighboring areas and nuclei are often striking. The variations among corlical areas may be surprising in the context of previous evidence that, except for primate area 17 ,

nucleus $(R)$. Bar, $200 \mu \mathrm{m}$. $C$, Cat-301 staining of the ventral posterior lateral $(V P L)$ and ventral posterior medial nuclei $(V P M)$ and the anterior pulvinar nucleus $(P l a)$. The cells in these nuclei are organized into clusters. $D$, Section adjacent to $C$ stained for anterogradely transported HRP following an injection into the principal nucleus of the trigeminal complex. By comparing the positions of the same blood vessels in the 2 sections $(x)$, the trigeminothalamic afferents are found to interdigitate with the clusters of Cat-301-positive cells in VPM. Bar, $200 \mu \mathrm{m}$ 
the numbers of neurons within an area (Rockel et al., 1980; Powell, 1981), the morphological types of neurons (Fairén et al., 1984), and probably the circuits that the neurons form (Jones, 1983a; Hendry, 1987) do not vary greatly among areas. Even the numbers of a well-defined neuronal subpopulation, the GABA-immunoreactive neurons, appear to remain constant across most areas of monkey cortex (Hendry et al., 1987). The homogeneity of the cortex has been accented in these studies, the implication being that afferent connections represent the major differences among areas. However, recent studies in which neurotransmitters, their related enzymes, and their receptors have been localized to cortical neurons have detected many differences among areas in the density and distribution of the neurons and their processes (Wise and Herkenham, 1982; Hendry et al., 1984c, 1987; Campbell et al., 1987; Jones et al., 1988). Such differences mark the borders between neighboring areas and are particularly prominent when areas of different lobes are compared. Staining with Cat-301 is thus a dramatic example of the general finding that when cortical neurons are chemically characterized, heterogeneity among areas becomes obvious. This heterogeneity is likely to represent differences in the organization of the cortical areas and may contribute to the physiological differences among areas.

Relatively little is known about differences in phenotypic expression among neurons of the dorsal thalamus. The same basic circuit elements exist in each nucleus of the cat and monkey thalamus (Jones, 1983b); these include one or more systems of ascending afferent axons, the relay neurons, and, in most species, a population of intrinsic neurons. Cat-301 stains the relay neurons of only a few nuclei. Relay neurons exist in several morphologically distinct forms within a single thalamic nucleus (Guillery, 1966; Friedlander et al., 1981; Yen and Jones, 1983), but the neurotransmitter properties of these cells and any chemical differences between neurons of different nuclei are unclear. Variations among thalamic nuclei include not only the sizes and packing densities of neurons, which serve to distinguish the structure of one nucleus from its neighbors, but also the sources of afferent axons and the targets of efferent axons (Jones, 1983b). The present study of Cat-301 staining and a previous study of $\mathrm{mAB}$ immunostaining (Levitt, 1984) provide evidence that relay cells in one nucleus differ from those of other nuclei in an immunocytochemically detectable fashion. The association of thalamic Cat-301 with relay cells projecting only to heavily stained cortical areas suggests that differences in staining among thalamic nuclei may be related to the differences in connections.

Differences among species. Cat-301 stains neurons in the cerebral cortex and thalamus of all the mammalian species studied. These findings indicate that, for all these species, at least some thalamic and cortical neurons express one or more molecules with a common epitope. Conservation of epitopes across species has been described in other studies (e.g., Venter et al., 1984), and highlighted by the observation that $\mathrm{mABs}$ raised against the eye of Drosophila recognize antigens that are also present in the human CNS (Miller and Benzer, 1983). More striking and possibly more significant are the differences in Cat301 staining of cortical and thalamic cells that exist across species. These differences include variations in the number and distribution of immunostained cells and the intensity of their staining. Such variations are appreciable between cats and monkeys, but are relatively modest when compared with the marked differences found between these species and rats, guinea pigs, and bush babies. The species differences raise the possibility that either a molecule similar in function and cell-surface location to the antigen(s) recognized by Cat-301 is present on many neurons, but is modified in some species so that the $\mathrm{mAB}$ fails to recognize it, or that the Cat-301 antigen(s) and function(s) are features of fewer cortical and thalamic neurons in some species.

Immunostaining of organized groups of neurons. Neurons of the cerebral cortex and of the LGN are organized into layers. Within a given layer, cells with similar connections and physiological properties are aggregated together. Through differences in the numbers of neurons stained and the intensity of their staining, Cat-301 identifies the laminar organization of the cerebral cortex and LGN of cats and monkeys. Within the cortex, the stained cells may be laminated because groups of connectionally specific neurons express the Cat-301 antigen (see below); in the LGN, a closer association appears to exist between Cat301 immunostaining and a neuron's functional properties. The greatest number of stained cells in the cat and monkey LGN is found in layers and subnuclei in which the well-known Y-type cell is found (Hockfield et al., 1983; Hendry et al., 1984a). $\mathrm{Y}$-type cells can be distinguished from other geniculate relay cells by their nonlinear spatial summation of visual stimuli (Enroth-Cugcll and Robson, 1966). In cats, such cells dominate the MIN population and, in the laminar dorsal LGN, dominate lamina $\mathrm{C}$ and form the largest cells of the $\mathrm{A}$ laminae (Cleland et al., 1971, 1976; Fukuda and Stone, 1974; Wilson and Stone, 1975; Wilson et al., 1976; Kratz et al., 1978; Dreher and Sefton, 1979). In monkeys, although criteria differ (So and Shapley, 1981), there is a consensus that neurons physiologically similar to the cat Y-type cell are the major cell population of the magnocellular laminae (Dreher et al., 1976; Sherman et al., 1976; Schiller and Malpeli, 1978). In all of these sites Cat-301 stains many neurons.

Neurons in the VPM nucleus of monkeys are clustered together into functional units in which groups of relay cells have receptive fields related to the same part of the face or mouth and project to isolated columns in the cortex (Jones and Friedman, 1982; Jones et al., 1986). The units, or "rods," are narrow and elongated in the anteroposterior dimension. Immunostaining with Cat-301 independently corroborates the organization of VPM neurons into rods, and shows that the relay neurons projecting to SI lie outside the zones of afferent terminations from the contralateral principal trigeminal nucleus. It is not known whether a second afferent source, possibly the ipsilateral principal trigeminal nucleus (Ganchrow and Mehler, 1986), selectively innervates the Cat-301-stained rods. In addition, it is not known what type of VPM neuron, left unstained by Cat301 , occupies the 7ones of contralateral trigeminal input. Since double-labeling studies indicate that the great majority of VPM neurons projecting to SI are stained by Cat-301, but that all GABA-positive neurons are unstained, the contralateral principal trigeminal nucleus may terminate among relay cells projecting to areas other than SI or among GABA cells intrinsic to VPM.

Columnar organization of Cat-301-positive neurons in monkey visual cortex. Cat-301 staining in certain layers of the monkey visual cortex coincides with well-known patterns of histochemical staining. In area 17, Cat-301-stained patches fall within narrow columnlike zones of $\mathrm{CO}$ staining that run vertically though the cortex at the centers of ocular dominance columns (Horton and Hubel, 1981; Livingstone and Hubel, 1982, 1984a). CO-staincd pcriodicitics are most prominent in layers II-III and 
VI and are in vertical register with one another and with similar, lighter-stained periodicities in layers IVB and V. Narrow, vertical columns of CO staining thus tend to traverse the thickness of area 17, being interrupted only by the homogeneous $\mathrm{CO}$ staining in layers IVA and IVC. In the present study, dense clusters of neurons were stained in layers III, IVB, and VI, and these were found to coincide with the patches of intense $\mathrm{CO}$ staining.

Electrophysiological studies and studies of ${ }^{14} \mathrm{C}$-2-deoxy-D-glucose uptake show that neurons within the $\mathrm{CO}$ periodicities of layers II-III lack orientation selectivity (Humphrey and Hendrickson, 1983; Livingstone and Hubel, 1984a), but are sensitive to specific wavelengths of light (Livingstone and Hubel, 1984a). The CO patches in layers II-III appear to be connected preferentially with one another (Livingstone and Hubel, 1984b) and with certain of the CO-stained bands that are present in area 18 (Livingstone and Hubel, 1983). The patches may receive at least part of their geniculocortical inputs from a specific population of LGN neurons that lie in the $S$ layers and in the interlaminar zones (Livingstone and Hubel, 1982). These physiological and anatomical studies suggest that the CO-stained patches in layers II-III are structurally and functionally distinct units within area 17 . The present study shows that within these units are clusters of cells that share cell-surface molecules recognized by Cat-301, indicating that cells within the units are also biochemically distinct.

The CO patches in layers II-III can be divided on physiological grounds into a central core and a surrounding shell (Livingstone and Hubel, 1984a). The core is made up of cells that display no selectivity for the orientation of a visual stimulus, whereas in the shell, cells display some orientation selectivity (Livingstone and Hubel, 1984a). In the present study, we found that for the patches in layers II-III, the core regions contain most of the Cat-301-positive cells. The CO patches in layers IIIII are also regions in which dense clusters of large punctuate profiles, probably representing axon terminals, are immunoreactive for the GABA-synthesizing enzyme glutamate decarboxylase (GAD; Hendrickson et al., 1981); but the GAD-positive somata in these layers are no denser in the patches than they are between the patches (Fitzpatrick et al., 1983; Hendry et al., 1987). Thus, the present findings in which GABA and Cat-301 were colocalized indicate that the Cat-301 staining in layers II-III and IVB is patchy because the $\mathrm{mAB}$ stains preferentially that subpopulation of GABA neurons situated at the core of the $\mathrm{CO}$ patches.

Only a fraction of the neurons within a $\mathrm{CO}$ patch are Cat301-positive, suggesting that the stained and unstained neurons in a patch may be fundamentally different types of cells. Three physiologically distinct classes of neuron have been identified in the patches (Livingstone and Hubel, 1984a). Two are colorsensitive, double-opponent neurons with different spectral sensitivities (red-green and yellow-blue), and the third is a type of broadband cell with a center-surround organization (Livingstone and Hubel, 1984a). One possibility is that Cat-301 preferentially stains one of these groups. The distribution of Cat301 -positive neurons in the magnocellular layers of the monkey LGN suggests that some are Y-type neurons (see above). A similar suggestion has been made (Hockfield et al., 1983; Hendry et al., 1984a) for neurons in the cat LGN because of their size and concentration in laminae $\mathrm{A}, \mathrm{Al}, \mathrm{C}$, and the MIN, and their projection to area 18 (Sur et al., 1984). There may be reason, then, to expect that the population of neurons in the $\mathrm{CO}$ patches stained with Cat-301 will prove to be responsive to broadband stimuli. However, the presence of Cat-301-positive neurons throughout the parvocellular layers of the LGN and their very limited numbers in layer IVC $\alpha$ of area 17 demonstrate that staining with this $\mathrm{mAB}$ is neither restricted to $\mathrm{Y}$-type cells nor includes all Y-type cells.

In area 18 of monkeys, $\mathrm{CO}$ and Cat-301 both stain a series of parallel bands. The $\mathrm{CO}$ staining consists of thick $(1-\mathrm{mm}$ wide) and thin (200-300- $\mu \mathrm{m}$-wide) bands that are oriented perpendicular to the border of areas 17 and 18 (Livingstone and Hubel, 1982; Tootell et al., 1983). The CO-rich bands in area 18 form specific interconnections with the CO-rich patches in area 17 (Livingstone and Hubel, 1983), and the bands and patches are both labeled by ${ }^{14} \mathrm{C}$-2-deoxy-D-glucose uptake when a diffuse, nonoriented visual stimulus is presented to the monkey at low spatial frcquencics (Tootcll ct al., 1983). Both are left unlabeled when an oriented stimulus is presented at high spatial frequencies (Tootell et al., 1983). Results of the present study (see also DeYoe et al., 1986) indicate that, in addition to their interconnections and functional similarities, neurons in the $\mathrm{CO}$ rich bands of area 18 and those in CO-rich patches in layers III and IVB of area 17 also display similar molecular characteristics, since a relatively large proportion are Cat-301-positive.

Repeating units of cortical organization have not been detected by $\mathrm{CO}$ staining in areas outside the monkey visual cortex. Cat-301 staining in the cat visual cortex and in many other areas of cat and monkey cortex also showed little evidence of a regular, periodic pattern. The somewhat patchy distribution of Cat-301positive neurons in the sensory-motor area and in other areas of monkey and cat cortex may be due to the selective staining of particular classes of efferent neurons, since these are often loosely grouped into irregular clusters (e.g., Jones and Wise, 1977; Murray and Coulter, 1981). However, the apparent failure of Cat-301 staining to detect ocular dominance columns in the cat and to recognize columnar arrangements associated with commissural and corticocortical fibers that have been demonstrated in many areas (e.g., Jones et al., 1975, 1979; GoldmanRakic and Schwartz, 1982) indicates that the $\mathrm{mAB}$ is not a general marker of cortical "columns."

\section{Common properties of Cat-301-positive neurons}

To determine whether Cat-301 recognizes groups of cells that, although present through much of the thalamus and cortex, are still interrelated, we compared the structure, connectivity, and neurotransmitter expression of groups of these cells. Cat-301positive neurons in areas 17 and 18 of the monkey cortex, in the VPM and VPL of the monkey thalamus, and in the LGNs of monkey and cat thalamus were clustered into orderly arrays. Although this finding cannot be generalized to the neocortex and thalamus as a whole (see above), the ability of Cat-301 staining to demonstrate the patterns of organization in these regions, as well as in the hippocampal formation, caudate nucleus, and olfactory cortex (unpublished observations) suggests that certain features of forebrain organization may be associated with the antigen(s) recognized by Cat- 301 .

Many Cat-301-positive neurons and groups of neurons in the cat and monkey cortex and thalamus are interconnected. At its broadest, the relationship between connectivity and immunostaining can be seen for the thalamus as a whole, since only those thalamic nuclei reciprocally connected with intensely stained areas of cortex contain Cat-301-positive neurons. At a finer level, for subpopulations of cells within a cortical area, 
Cat-301 staining may also be related to connectivity. Thus, of the many types of target-specific pyramidal neurons in the sensory-motor areas of the monkey cortex (Jones and Wise, 1977), those projecting to the spinal cord are stained preferentially. A certain specificity may also exist for the connections of Cat-301positive GABA neurons. In layer $V$ of the sensory-motor areas of monkey cortex, the large basket cell, which synapses upon the somata and dendrites of the stained corticospinal cells (DeFelipe et al., 1986), is Cat-301-positive, while many smaller GABA cells of the same layer are unstained by Cat-301. These data suggest that within a very large population of thalamic and cortical neurons, there are groups of Cat-301-positive cells that are closely related by their connectivity. The question can be asked, therefore, whether the antigen(s) recognized by Cat-301 contribute to the biological processes that underlie establishment or maintenance of connections.

Neuronal cell-surface molecules have been implicated in many aspects of nervous system development, including cell-cell recognition (Barondes and Rosen, 1976) and adhesion (Edelman, 1984), and in neurotransmission between adult central neurons. As in previous studies (Hockfield and McKay, 1983a), we found that the antigen(s) recognized by Cat-301 lies along the surfaces of cortical and thalamic neurons, possibly within the extracellular matrix. Two features stand out from this and previous studies of Cat-301: it stains neurons throughout the neuraxisthus the Cat-301 antigen(s) may be involved with neuronal events that occur in many regions of the central nervous system; and it stains only a subpopulation of neurons in any one region, suggesting that the antigen(s) may function selectively to distinguish among cells within an aggregrate. The localization studies have identified a provocative distribution of immunocytochemically characterized neurons. The nature of the Cat-301 antigen(s) and their functional role in the nervous system are the subjects of studies currently in progress (Zaremba et al., 1985).

\section{References}

Arimatsu, Y., J. R. Naegle, and C. J. Barnstable (1987) Molecular markers of neuronal subpopulations in layers 4,5 and 6 of cat primary visual cortex. J. Neurosci. 7: 1250-1263.

Barnstable, C. J. (1980) Monoclonal antibodies which recognize different cell types in the rat retina. Nature 286: 231-235.

Barondes, S. H., and S. D. Rosen (1976) Cell surface carbohydratebinding proteins: Role in cell recognition. In Neuronal Recognition, S. H. Barondes, ed., pp. 331-356, Plenum, New York.

Berman, N., and E. G. Jones (1977) A retino-pulvinar projection in the cat. Brain Res. 134: 237-248.

Campbell, M. J., D. A. Lewis, R. Benoit, and J. H. Morrison (1987) Regional heterogeneity in the distribution of somatostatin-28- and somatostatin -28 J. Neurosci. 7: 1133-1144.

Celio, M. R. (1986) Parvalbumin in most $\gamma$-aminobutyric acid-containing neurons of the rat cerebral cortex. Science 231: 995-997.

Cheney, P. D., and E. E. Fetz (1980) Functional classes of primate corticomotoneuronal cells and their relation to active force. J. Neurophysiol. 44: 773-791.

Chikaraishi, D. M., M. H. Brilliant, and E. J. Lewis (1983) Cloning and characterization of rat-brain-specific transcripts: Rare, brain-specific transcripts and tyrosine hydroxylase. Cold Spring Harbor Symp. Quant. Biol. 48: 309-318.

Cleland, B. G., M. W. Dubin, and W. R. Levick (1971) Sustained and transient neurones in the cat's retina and lateral geniculate nucleus. J. Physiol. (Lond.) 217: 473-496.

Cleland, B. G., W. R. Levick, R. Morstyn, and H. G. Wagner (1976) Lateral geniculate relay of slowly conducting retinal afferents to the cat visual cortex. J. Physiol. (Lond.) 225: 299-320.

DeFelipe, J., S. H. C. Hendry, and E. G. Jones (1986) A correlative electron microscopic study of basket cells and large GABAergic neu- rons in the monkey sensory-motor cortex. Neuroscience 17:9911009.

DeOlmos, J., H. Hardy, and L. Heimer (1978) The afferent connections of the main and accessory olfactory bulb formations in the rat: An experimental HRP study. J. Comp. Neurol. 181: 213-241.

DeYoe, E. A., H. Garren, S. Hockfield, and D. Van Essen (1986) Cat301 antibody identifies distinct areas and subdivisions in macaque extrastriate cortex. Soc. Neurosci. Abstr. 12: 130.

Dreher, B., and A. J. Sefton (1979) Properties of neurons in cat's dorsal lateral geniculate nucleus: A comparison between medial interlaminar and laminated parts of the nucleus. J. Comp. Neurol. 183: 47-64.

Dreher, B., Y. Fukuda, and R. W. Rodieck (1976) Identification, classification and anatomical segregation of cells with $\mathrm{X}$-like and $\mathrm{Y}$-like properties in the lateral geniculate nucleus of Old World primates. J. Physiol. (Lond.) 258: 433-452.

Edelman, G. M. (1984) Modulation of cell adhesion during induction, histogenesis and perinatal development of the nervous system. Annu. Rev. Neurosci. 7: 339-377.

Emson, P. C., and S. P. Hunt (1984) Peptide-containing neurons of the cerebral cortex. In Cerebral Cortex, vol. 2, E. G. Jones and A. Peters, eds., pp. 145-169, Raven, New York.

Enroth-Cugell, C. C., and J. G. Robson (1966) The contrast sensitivity of retinal ganglion cells of the cat. J. Physiol. (Lond.) 187: 517-534.

Evarts, E. E., C. Fromm, J. Kroller, and V. A. Jennings (1981) Motor cortex control of finely graded forces. J. Neurophysiol. 49: 1199-1215.

Fairén, A., J. DeFelipe, and J. Regidor (1984) Nonpyramidal neurons. General account. In Cerebral Cortex, vol. 1, A. Peters and E. G. Jones, eds., pp. 201-253, Plenum, New York.

Fitzpatrick, D., J. S. Lund, and D. Schmechel (1983) Glutamic acid decarboxylase immunoreactive neurons and terminals in the visual cortex of the cat and monkey. Soc. Neurosci. Abstr. 9: 616.

Fitzpatrick, D., G. R. Penny, and D. E. Schmechel (1984) Glutamic acid decarboxylase-immunoreactive neurons and terminals in the lateral geniculate nucleus of the cat. J. Neurosci. 4: 1809-1829.

Freund, T. F., K. A. C. Martin, A. D. Smith, and P. Somogyi (1983) Glutamate decarboxylase-immunoreactive terminals of Golgi-impregnated axoaxonic cells and of presumed basket cells in synaptic contact with pyramidal neurons of the cat's visual cortex. J. Comp. Neurol. 221: 263-278

Friedlander, M. J., C.-S. Lin, L. R. Stanford, and S. M. Sherman (1981) Morphology of functionally identified neurons in lateral geniculate nucleus of the cat. J. Neurophysiol. 46: 80-129.

Fry, K. R., D. Travella, Y. Y. T. Su, Y. W. Peng, C. B. Watt, and D. M. K. Lam (1985) A monoclonal antibody specific for retinal ganglion cells of mammals. Brain Res. 338: 360-365.

Fukuda, Y., and J. Stone (1974) Retinal distribution and central projections of $Y_{-}, X_{-}$, and W-cells of the cat's retina. J. Neurophysiol. 37: 749-772.

Ganchrow, D., and W. R. Mehler (1986) Dual efferent projections of the trigeminal principal sensory nucleus to the thalamic ventroposteromedial nucleus in the squirrel monkey. Neurosci. Lett. 68: 181186.

Gilbert, C. D. (1977) Laminar differences in receptive field properties of cells in cat primary visual cortex. J. Physiol. (Lond.) 268: 391421

Gilbert, C. D., and J. P. Kelly (1975) The projections of cells in different layers of the cat's visual cortex. J. Comp. Neurol. 163: 81106.

Gilbert, C. D., and T. N. Wiesel (1979) Morphology and intracortical projections of functionally characterised neurones in the cat visual cortex. Nature 280:120-125.

Goldman-Rakic, P. S., and M. L. Schwartz (1982) Interdigitation of contralateral and ipsilateral columnar projections to frontal association cortex in primates. Science 216: 755-758.

Golgi, C. (1883) Recherches sur l'histologie des centres nerveux. Arch. Ital. Biol. 4: 92-123.

Guillery, R. W. (1966) A study of Golgi preparations from the dorsa lateral geniculate nucleus of the cat. J. Comp. Neurol. 128: 21-50.

Guillery, R. W., E. E. Guisert, Jr., E. H. Polley, and C. A. Mason (1980) An analysis of the retinal afferents to the cat's medial interlaminar nucleus and its rostral thalamic extension, the "geniculate wing." $\mathrm{J}$. Comp. Neurol. 194: 117-142.

Hahn, W. E., N. Chaudhari, L. Beck, K. Wilber, and D. Peffley (1983) Genetic expression and postnatal development of the brain: Some 
characteristics of nonpolyadenylated mRNA's. Cold Spring Harbor Symp. Quant. Biol. 48: 465-476.

Hawkes, R., E. Niday, and A. Matus (1982) Monoclonal antibodies identify novel neural antigens. Proc. Natl. Acad. Sci. USA 79: 24102414

Hawkes, R., M. Colonnier, and N. Leclerc (1985) Monoclonal antibodies reveal sagittal banding in the rodent cerebellar cortex. Brain Res. 333: 359-365.

Hendrickson, A. E., S. P. Hunt, and J.-Y. Wu (1981) Immunocytochemical localization of glutamic acid decarboxylase in monkey striate cortex. Nature 292: 605-607.

Hendry, S. H. (1987) Recent advances in understanding the intrinsic circuitry of the cerebral cortex. In Higher Brain Functions: Recent Explorations of the Brain's Emergent Properties, S. P. Wise, ed., pp. 241-283, Wiley, NY.

Hendry, S. H. C., S. J. Hockfield, R. D. G. McKay, and E. G. Jones (1983a) Features of the organization of macaque monkey visual cortex demonstrated with monoclonal antibody CAT 301 . Soc. Neurosci. Abstr. 9: 39

Hendry, S. H. C., C. R. Houser, E. G. Jones, and J. E. Vaughn (1983b) Synaptic organization of immunocytochemically identified GABA neurons in the monkey sensory-motor cortex. J. Neurocytol. 12:639660.

Hendry, S. H. C., S. Hockfield, E. G. Jones, and R. McKay (1984a) Monoclonal antibody that identifies subsets of neurons in the central visual system of monkey and cat. Nature 307: 267-269.

Hendry, S. H. C., E. G. Jones, J. DeFelipe, C. Brandon, and P. C. Emson (1984b) Neuropeptide-containing neurons of the cerebral cortex are also GABAergic. Proc. Natl. Acad. Sci. USA 81: 6526-6530.

Hendry, S. H. C., E. G. Jones, and P. C. Emson (1984c) Morphology, distribution and synaptic relations of somatostatin- and neuropeptide $\mathrm{Y}$-immunoreactive neurons in rat and monkey neocortex. J. Neurosci. 4: 2497-2517.

Hendry, S. H. C., H. D. Schwark, E. G. Jones, and J. Yan (1987) Numbers and proportions of GABA-immunoreactive neurons in different areas of monkey cerebral cortex. J. Neurosci. 7: 1503-1519.

Hockfield, S., and R. D. G. McKay (1983a) A surface antigen expressed by a subset of neurons in the vertebrate central nervous system. Proc. Natl. Acad. Sci. USA 80: 5758-5761.

Hockfield, S., and R. McKay (1983b) Monoclonal antibodies demonstrate the organization of axons in the leech. J. Neurosci. 3: 369375.

Hockfield, S., R. D. McKay, S. H. C. Hendry, and E. G. Jones (1983) A surface antigen that identifies ocular dominance columns in the visual cortex and laminar features of the lateral geniculate nucleus. Cold Spring Harbor Symp. Quant. Biol. 48: 877-889.

Horton, J. C.. and D. H. Hubel (1981) Regular patchy distribution of cytochrome oxidase staining in primary visual cortex of macaque monkey. Nature 292: 762-764.

Houser, C. R., J. E. Vaughn, R. P. Barber, and E. Roberts (1980) GABA neurons are the major cell type of the nucleus reticularis thalami. Brain Res. 200: 341-354.

Houser, C. R., S. H. C. Hendry, E. G. Jones, and J. E. Vaughn (1983) Morphological diversity of immunocytochemically identified GABA neurons in the monkey sensory-motor cortex. J. Neurocytol. 12:617638.

Houser, C. R., J. E. Vaughn, S. H. C. Hendry, E. G. Jones, and A. Peters (1984) GABA neurons in the cerebral cortex. In Cerebral Cortex, vol. 2, E. G. Jones and A. Peters, eds., pp. 63-89, Plenum, New York.

Hubel, D. H., and T. N. Wiesel (1962) Receptive fields, binocular interaction and functional architecture in the cat's visual cortex. J. Physiol. (Lond.) 160: 106-154.

Hubel, D. H., and T. N. Wiesel (1968) Receptive fields and functional architecture of monkey striate cortex. J. Physiol. (Lond.) 195: 215 243.

Humphrey, A. L., and A. E. Hendrickson (1983) Background and stimulus-induccd patterns of high metabolic activity in the visual cortex (area 17) of the squirrel and macaque monkey. J. Neurosci. 3: 345-358.

Johansen, J., I. Thompson, R. R. Stewart, and R. D G. McKay (1985) Expression of surface antigens recognized by the monoclonal antibody Lan 3-2 during embryonic development in the leech. Brain Res. 343: $1-7$.

Jones, E. G. (1975) Varieties and distribution of non-pyramidal cells in the somatic sensory cortex of the squirrel monkey. J. Comp. Neurol. 160: 205-268.
Jones, E. G. (1983a) Identification and classification of intrinsic circuit elements in the neocortex. In Dynamic Aspects of Neocortical Function, G. Edelman, W. M. Cowan, and W. E. Gall, eds., pp. 7-40, Wiley, New York.

Jones, E. G. (1983b) Organization of the thalamocortical complex and its relation to sensory processes. In Handbook of Physiology-The Nervous System III, I. Darian-Smith, ed., pp. 149-212, American Physiological Society, Washington, D.C.

Jones, E. G. (1985) The Thalamus, Plenum, New York.

Jones, E. G., and D. P. Friedman (1982) Projection pattern of functional components of thalamic ventrobasal complex on monkey somatosensory cortex. J. Neurophysiol. 48: 521-544.

Jones, E. G., and S. H. C. Hendry (1986) Peptide-containing neurons of the primate cerebral cortex. In Neuropeptides in Neurologic and Psychiatric Disease, J. B. Martin and J. D. Barchas, eds., pp. 163178, Raven, New York.

Jones, E. G., and S. P. Wise (1977) Size, laminar and columnar distribution of efferent cells in the sensory-motor cortex of monkeys. $J$. Comp. Neurol. 175: 391-438.

Jones, E. G., H. Burton, and R. Porter (1975) Commissural and cortico-cortical "columns" in the somatic sensory cortex of primates. Science 190: 572-574.

Jones, E. G., J. D. Coulter, and S. P. Wise (1979) Commissural columns in the sensory-motor cortex of monkeys. J. Comp. Neurol. 188 . 113-136.

Jones, E. G., R. D. G. McKay, S. J. Hockfield, and S. H. C. Hendry (1983) Differential distribution of neurons immunoreactive to monoclonal antibody CAT 301 in the mammalian cerebral cortex. Soc. Neurosci. Abstr. 9: 39.

Jones, E. G., S. H. C. Hendry, and C. Brandon (1986) Cytochrome oxidase staining reveals functional organization of monkey somatosensory thalamus. Exp. Brain Res. 62: 438-442.

Jones, E. G., J. DeFelipe, S. H. C. Hendry, and J. E. Maggio (1988) A study of tachykinin immunoreactive neurons in monkey cerebral cortex. J. Neurosci. (in press).

Kölliker, A. von (1896) Handbuch der Gewehelehre des Menschen vol. 2; Nervensystem des Menschen und der Thiere, Engelman, Leipzig.

Kratz, K. E., S. V. Webb, and S. M. Sherman (1978) Electrophysiological classification of $\mathrm{X}$ - and $\mathrm{Y}$-cells in the cat's lateral geniculate nucleus. Vision Res. 18: 489-492.

Lemmon, V., and D. I. Gottlieb (1982) Monoclonal antibodies selective for the inner portion of the chick retina. J. Neurosci. 2: 531-535.

LeVay, S., D. H. Hubel, and T. N. Wiesel (1975) The pattern of ocular dominance columns in macaque visual cortex revealed by a reduced silver stain. J. Comp. Neurol. 159: 559-576.

Levitt, P. (1984) A monoclonal antibody to limbic system neurons. Science 233: 299-301.

Livingstone, M. S., and D. H. Hubel (1982) Thalamic inputs to cytochrome oxidase-rich regions in monkey visual cortex. Proc. Natl. Acad. Sci. USA 79: 6098-6101.

Livingstone, M. S., and D. H. Hubel (1983) Specificity of corticocortical connections in monkey visual system. Nature 304: 531-534.

Livingstone, M. S., and D. H. Hubel (1984a) Anatomy and physiology of a color system in the primate visual cortex. J. Neurosci. 4: 309356.

Livingstone, M. S., and D. H. Hubel (1984b) Specificity of intrinsic connections in primate primary visual cortex. J. Neurosci. 4: 2830 2835.

Lund, J. S. (1973) Organization of neurons in the visual cortex, area 17, of the monkey (Macaca mulatta). J. Comp. Neurol. 147: 455496.

Lund, J. S., R. D. Lund, A. E. Hendrickson, A. H. Bunt, and A. F. Fuchs (1975) The origin of efferent pathways from the primary visual cortex (area 17) of the macaque monkey as shown by the retrograde transport of horseradish peroxidase. J. Comp. Neurol. 164: 287-304.

Macchi, G., M. Bentivoglio, M. Molinari, and D. Minciacchi (1984) The thalamo-caudate versus thalamo-cortical projections as studied in the cat with fluorescent retrograde double labeling. Exp. Brain Res. 54: 225-239.

Marin-Padilla, M. (1969) Origin of the pericellular baskets of the pyramidal cells of the human motor cortex: A Golgi study. Brain Res. 14: 633-646.

Martin, K. A. C., and D. Whitteridge (1984) Form, function and intracortical projections of spiny neurones in the striate cortex of the cat. J. Physiol. (Lond.) 353: 463-504. 
McKay, R. D. G., and S. J. Hockfield (1982) Monoclonal antibodies distinguish antigenically discrete neuronal types in the vertebrate central nervous system. Proc. Natl. Acad. Sci. USA 79: 6747-6751.

McKay, R. D., S. Hockfield, J. Johanses, and K. Fredericksen (1983) The molecular organization of the leech nervous system. Cold Spring Harbor Symp. Quant. Biol. 48: 599-610.

Miller, C. A., and S. Benzer (1983) Monoclonal antibody cross-reactions between Drosophila and human brain. Proc. Natl. Acad. Sci. USA 80: 7641-7645.

Montero, V. M., and J. Zempel (1985) Evidence for two types of GABA-containing interneurons in the $\Lambda$-laminae of the cat lateral geniculate nucleus: A double-label HRP and GABA-immunocytochemical study. Exp. Brain Res. 60: 603-609.

Murray, E. A., and J. D. Cuulter (1981) Organization of corticospinal neurons in the monkey. J. Comp. Neurol. 195: 339-365.

Nakagawa, F., B. A. Schulte, J.-Y. Wu, and S. S. Spicer (1986) GABAergic neurons of rodent brain correspond partially with those staining for glycoconjugate with terminal $\mathrm{N}$-acetylgalactosamine. J. Neurocytol. 15: 389-396.

Oertel, W. H., A. M. Graybiel, E. Mugnaini, R. P. Elde, D. E. Schmechel, and I. J. Kopin (1983) Coexistence of glutamic acid decarboxylaseand somatostatin-like immunoreactivity in neurons of the feline nucleus reticularis thalami. J. Neurosci. 3: 1322-1332.

Parnavelas, J. G., R. A. Burne, and C.-S. Lin (1983) Distribution and morphology of functionally identified neurons in the visual cortex of the rat. Brain Res. 261: 21-29.

Penny, G. R., D. Fitzpatrick, D. E. Schmechel, and I. T. Diamond (1983) Glutamic acid decarboxylase-immunoreactive neurons and horseradish peroxidase-labeled projection neurons in the ventral posterior nucleus of the cat and Galago senegalensis. J. Neurosci. 3: 18681887.

Peters, A., and E. G. Jones (1984) Classification of cortical neurons In Cerebral cortex vol. 1, A. Peters and E. G. Jones, eds., pp. 107122, Plenum, New York.

Peters, A., and J. Regidor (1981) A reassessment of the forms of nonpyramidal neurons in area 17 of cat visual cortex. J. Comp. Neurol. 203: $685-716$.

Powell, T. P. S. (1981) Certain aspects of the intrinsic organisation of the cerebral cortex. In Certain Aspects of the Intrinsic Organisation of the Cerebral Cortex, O. Pompeiano and C. Ajmone Marsan, eds., pp. 1-19, Raven, New York.

Ramón y Cajal, S. (1891) Sur la structure de l'écorce cérébrale de quelques mammiferes. Cellule $7: 125-176$

Retzius, M. G. (1893) Die Cajalschen Zellen der Grosshirnrinde beim Menschen und bei Säugethieren. Biol. Untersuch. N. F. 4: 1-9.

Ribak, C. E. (1978) Aspinous and sparsely spinous stellate neurons in the visual cortex of rats contain glutamic acid decarboxylase. J. Neurocytol. 7: 461-478.

Rockel, A. J., R. W. Hiorns, and T. P. S. Powell (1980) The basic uniformity in structure of the neocortex. Brain 103: 221-244.

Schiller, P. H., and J. G. Malpeli (1978) Functional specificity of lateral geniculate nucleus laminae of the rhesus monkey. J. Neurophysiol. 41: 788-797.

Schmechel, D. E., B. G. Vickrey, D. Fitzpatrick, and R. P. Elde (1984) GABAergic neurons of mammalian cerebral cortex: Widespread subclass defined by somatostatin content. Neurosci. Lett. 47: 227-232.

Sherman, S. M., J. R. Wilson, J. H. Kaas, and S. V. Webb (1976) Xand $Y$-cells in the dorsal lateral geniculate nucleus of the owl monkey (Aotus trivirgatus). Science 192: 475-477.

So, Y. T., and R. Shapley (1981) Spatial tuning of cells in and around lateral geniculate nucleus of the cat: $\mathrm{X}$ and $\mathrm{Y}$ relay cells and perigeniculate interneurons. J. Neurophysiol. 45: 107-120.

Somogyi, P., and I. Soltesz (1986) Immunogold demonstration of GABA in synaptic terminals of intracellularly recorded, horseradish peroxidase-filled basket cells in the cat's visual cortex. Neuroscience. 19: 1051-1065.

Somogyi, P., A. J. Hodgson, A. D. Smith, M. G. Nunzi, A. Gorio, and
J.-Y. Wu (1984) Different populations of GABAergic neurons in the visual cortex and hippocampus of cat contain somatostatin- or cholecystokinin-immunoreactive material. J. Neurosci. 4: 2590-2603.

Sternberger, L. A., L. W. Harwell, and N. Sternberger (1982) Neurotypy: Regional individuality in rat brain detected by immunocytochemistry with monoclonal antibodies. Proc. Natl. Acad. Sci. USA 79: $1326-1330$

Streit, P. (1984) Glutamate and aspartate as transmitter candidates in the cerebral cortex. In Cerebral Cortex, vol. 2, E. G. Jones and A. Peters, eds., pp. 119-143, Plenum, New York.

Sur, M., S. Hockficld, M. Macavoy, P. Garraghty, M. Kritzer, and R. McKay (1984) A monoclonal antibody that may identify Y-cells in the cat lateral geniculate nucleus. Soc. Neurosci. Abstr. 10: 106.

Tootell, R. B. H., M. S. Silverman, R. L. DeValois, and G. H. Jacobs (1983) Functional organization of the second cortical visual area in primates. Science 220:737-739.

Valverde, F. (1971) Short axon neuronal subsystems in the visual cortex of the monkey. Int. J. Neurosci. 1: 181-197.

Venter, J. C., B. Eddy, L. M. Hall, and C. M. Fraser (1984) Monoclonal antibodies detect the conservation of muscarinic cholinergic receptor structure from Drosophila to human brain and detect possible structural homology with alpha 1-adrenergic receptors. Proc. Natl. Acad. Sci. USA 81: 272-276.

Wamsley, J. K. (1984) Autoradiographic localization of receptor sites in the cerebral cortex. In Cerebral Cortex, vol. 2, E. G. Jones and A. Peters, eds., pp. 119-143, Plenum, New York.

White, E. L. (1986) Termination of thalamic afferents in the cerebral cortex. In Cerebral Cortex, vol. 5, E. G. Jones and A. Peters, eds., pp. 271-290, Plenum, New York.

Wiesel, T. N., D. H. Hubel, and D. M. K. Lam (1974) Autoradiographic demonstration of ocular dominance columns in the monkey striate cortex by means of transsynaptic transport. Brain Res. 79: 273-279

Wilson, P. D., and J. Stone (1975) Evidence of W-cell input to the cat's visual cortex via the $\mathrm{C}$ laminae of the lateral geniculate nucleus. Brain Res. 92: 472-478.

Wilson, P. D., M. H. Rowe, and J. Stone (1976) Properties of relay cells in cat's lateral geniculate nucleus: A comparison of W-cells with X-and Y-cells. J. Neurophysiol. 39: 1193-1209.

Wise, S. P., and M. Herkenham (1982) Opiate receptor distribution in the cerebral cortex of the rhesus monkey. Science 218: 387-389.

Wong-Riley, M. T. T. (1979) Changes in the visual system of monocularly sutured or enucleated cats demonstrable with cytochrome oxidase histochemistry. Brain Res. 171: 11-28.

Wood, J. N., L. Hudson, I. M. Jessell, and M. Yamamoto (1982) A monoclonal antibody defining antigenic determinants on subpopulations of mammalian neurones and Trypanosoma cruzi parasites. Nature 296: 34-38.

Yen, C.-T., and E. G. Jones (1983) Intracellular staining of physiologically identified neurons and axons in the somatosensory thalamus of the cat. Brain Res. 280: 148-154.

Yen, C.-T., M. Conley, S. H. C. Hendry, and E. G. Jones (1985) The morphology of physiologically identified GABAergic neurons in the somatic sensory part of the thalamic reticular nucleus in the cat. $J$. Neurosci. 5: 2254-2268.

Young, L. H. Y., and J. E. Dowling (1984) Monoclonal antibodies distinguish subtypes of retinal horizontal cells. Proc. Natl. Acad. Sci. USA 81: 6255-6259.

Zaremba, S., E. Waldvogel, and S. Hockfield (1985) Monoclonal antibody Cat-301: Recognition of a surface antigen and subsets of cells in human central visual areas and biochemical purification of the antigen from guinea pig brain. Soc. Neurosci. Abstr. 11: 1106.

Zipser, B. (1982) Complete patterns of neurons with characteristic antigens in the leech central nervous system. J. Neurosci. 2: 14531464.

Zipser, B., and R. McKay (1981) Monoclonal antibodies distinguish identifiable neurons in the leech. Nature 289: 549-554. 